\title{
Aerodynamics of aero-engine installation
}

\author{
Tomasz P Stańkowski ${ }^{1}$, David G MacManus1, Christopher TJ Sheaf², Robert Christie1,
}

\begin{abstract}
This paper describes current progress in the development of methods to assessaero-engine airframe installation effects. The aerodynamic characteristics of isolated intakes, a typical transonic transport aircraft as well as a combination of a through flow nacelle and aircraft configuration have been evaluated. The validation task for an isolated engine nacelle is carried out with concern for the accuracy in the assessment of intake performance descriptors such as mass flow capture ratio and drag rise Mach number. The necessary mesh and modelling requirements to simulate the nacelle aerodynamics are determined. Furthermore, the validation of the numerical model for the aircraft is performed as an extension of work that has been carried out under previous drag prediction research programmes. The validation of the aircraft model has been extended to include the geometry with throughflow nacelles. Finally, the assessment of the mutual impact of the through flow nacelle and aircraft aerodynamics was performed. The drag and lift coefficient breakdown has been presented in order to identify the component sources of the drag associated with the engine installation. The paper concludes with an assessment of installation drag for through-flow nacelles and the determination of aerodynamic interference between the nacelle and the aircraft.
\end{abstract}

\section{Keywords}

Engine-airframe integration, through-flow nacelle, aerodynamic interference, computational fluid dynamics, NASA Common Research Model

${ }^{1}$ Centre for Propulsion, Cranfield University, United Kingdom

${ }^{2}$ Rolls Royce plc., Derby, United Kingdom

Corresponding author:

David MacManus, Centre for Propulsion, Cranfield University, Cranfield, Bedfordshire, MK43 0AL, UK.

Email: D.G.MacManus@cranfield.ac.uk 


\section{Introduction}

To meet the expected future improvements in overall aircraft efficiency, it is necessary to ensure that the installation and integration of the engine with the airframe is properly assessed. The issue of installation is as old as turbojet engines and dates back to late 40 s and early $50 \mathrm{~s}$. To avoid difficulties of podded nacelles some concepts located the turbojet engine in the fuselage in case of fighters or in the wing, as for example the De Havilland Comet. However, the growth of engine by-pass ratio favoured the podded configuration, as it became impractical to embed the engines in the wing. Moreover, the cruise velocity of commercial aircraft continuously increased, thus the aerodynamic interference become of greater interest in early80s1. A range of experimental studies dedicated to the problem of podded nacelle installation under a swept transonic wing23indicated installation drag for two nacellesto be in the order of 75 aircraft drag counts (dc). The key parameters that affect the installation drag were defined as nacelle axial and vertical position and nacelle toe-in angle. The contribution of pylon drag was consideredthrough an estimate of viscous terms which was corrected by \pm 3 aircraft drag counts for the pressure drag2, based on the geometrical design of the pylon. Currently, the effect of engine installation for a typical conventional podded under-wing engine configuration is estimated to be in the order of 30 to 50 drag counts per two engines4although it is sensitive to the relative engine size and position on the wing $4,5,6$. The effect of engine size becomes significant for larger diameters 7.

The development of numerical tools and an increase in computational power led to a series of Drag Prediction Workshops, where the second series was dedicated to nacelle installation effects8. The success of the initial workshop triggered the design of the more modern aircraft geometry of the NASA Common Research Model9. The publication of substantial experimental datasets with and without through-flow nacelles ${ }^{10}{ }^{11}$ makesthe Common Research Model a benchmark validation activity for the assessment of installation effect.In this context, it is anticipated that engine installation will become an increasingly important concern as engine 
diameters are expected to increase in pursuit of improved propulsive efficiency. It is also expected that knowledge of these aspects at the preliminary design stage will become more important to facilitate timely and informed decisions on engine cycle, size and airframe integration. A key element of the development of future civil aircraft is a robust assessment of the mutual interactions, and therefore of the thrust and drag characteristics, of the combined engine and aircraft configuration. The aim of this research is to build on experience from the Drag Prediction Workshops, to develop computational tools to evaluate nacelle drag, and to assess the nacelle installation interference drag for a typical civil transport configuration. The long-term purpose is to create a framework that will evaluate the combined engine and aircraft configuration for a specified flight mission and that could be applied at a preliminary design stage. In this context, the current paper presents the drag assessments for nacelle installation, where the work was carried out with the use of the NASA Common Research Model with through-flow nacelles.

\section{Methods and scope}

A key focus of the current work is the use of computational fluid dynamics (CFD) to assess the aerodynamic characteristics for intake and nacelle drag, as well as for the evaluation of installation aerodynamic effects for a through-flow nacelle. These activities provide a solid validation for a broader project, for which the overall objective is to develop a framework that combines an engine thermodynamic model, aircraft performance method, engine installation aspects with a flight trajectory approach. The aerodynamics of installation is evaluated with use of three key computational building blocks such as computation of isolated nacelles, isolated airframe and the aircraft, as airframe with through-flow nacelles. It is the comparison between those three building blocks that allows for the assessment of aerodynamic interference and the decomposition of installation drag. 


\section{Test case configurations}

\section{Isolated nacelles.}

The validation of the computational method and the drag extraction approach for isolated nacelle configurations was based on the experimental test cases of Langley et al. ${ }^{12}$.Within this previous experimental work a total of six axisymmetric cowls were investigated. Two of the key cowl designs from this dataset have been selected for the current validation study and will be referred to as in the original work ${ }^{12}$ as Cowl 1 and Cowl 3 . The rationale within broader context(Table 1 ) is to assess the capability of numerical methods in determination of drag characteristics for nacelles. Both cowls havethe same maximum diameter $\left(D_{\max }\right)$. Their intake geometries are characterized by a contraction ratio of $C R=1.25$, where contraction ratio is defined as the ratio of highlight area to intake throat area $\left(A_{h i} / A_{t h}\right)$. In both configurations the proportion of highlight diameter to maximum diameter is $D_{h i} / D_{\max }=0.85$ and the proportion of the forebody length to the maximum diameter is $L_{F} / D_{\max }=0.45$. Relative to Cowl 1 , Cowl 3 is designed with a notably decreased leading edge radius from $R_{L E} / D_{\max }=0.0224$ to $R_{L E} / D_{\max }=0.014$ and an increased curvature near the nacelle crest was used. Within the current study, the aim was to choose two cowls which are representative of two different design philosophies to assess the CFD for a range of possible flow conditions. Based on the experimental results, for this validation work, Cowl 1 was chosen due to its higher drag rise Mach number of $M_{D R}=0.846$ but with a higher baseline drag of $C_{D \text { nac }}=0.0375$ for operating conditions of a freestream Mach number $\left(M_{\infty}\right)$ of 0.7 and a massflow capture ratio (MFCR) of 0.7 , where MFCR is the ratio of the flow area of pre-entry streamtube at upstream infinity $\left(A_{\infty}\right)$ divided by a geometrical intake area $\left(A_{h i}\right)$. It was chosen to compare it with Cowl 3 which has an inferior performance in terms of drag rise $\left(M_{D R}=0.819\right)$ but better design characteristicsunder spillage conditions, with a drag coefficient $C_{D \text { nac }}=0.047$ compared to $C_{D \text { nac }}=0.053$ for Cowl 1 , with both evaluated at $M_{\infty}=0.85$ and MFCR $=0.55$.

The experimental tests ${ }^{12}$ were performed at subsonic and transonic conditions across a freestream Mach number $\left(M_{\infty}\right)$ range of 0.4 to 0.95 and with an intake MFCR variation between 
0.2and 0.9. These tests were conducted at a constant intake incidence of zero and therefore an axisymmetric computational model can be applied. A two dimensional axisymmetric circular domain (Figure 1)has been established with a far-field boundary condition located at a distance of approximately 60 nacelle maximum diameters $\left(D_{\max }\right)$. The $60 D_{\max }$ was determined from a domain independence study which evaluated the effect of domain sizes from $60 D_{\max }$ to $150 D_{\max }$. This study showed that for a typical nacelle configuration at $M_{\infty}=0.85$ and for MFCR across the range of 0.4 to 0.75 , that a domain of $60 D_{\max }$ was sufficiently large to ensure no spurious effects on the flow field.For example, for Cowl 1, the increase in $C_{D \text { nac }}$ for a domain of $150 D_{\max }$ at typical conditions of $M_{\infty}=0.85$ and MFCR $=0.7$ increased the drag coefficient by less than $0.1 \%$.

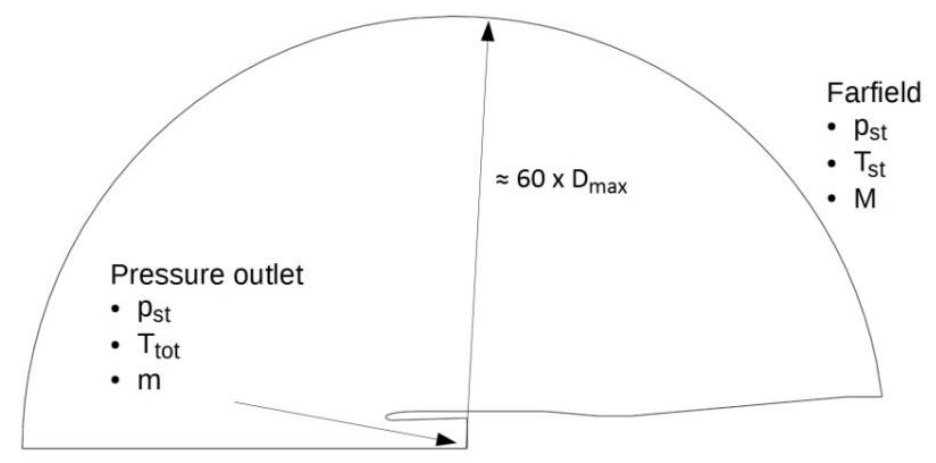

Figure 1. The domain and boundary conditions for the isolated nacelle investigation for the ARA cowls $^{12}$

The pressure far-field boundary condition(Figure 1)has been used in the simulations, which allowed for the specification of the free stream Mach number, static pressure and static temperature. Moreover, a pressure outlet boundary condition was assigned at the end of the internal duct in order to control the intake mass-flow and therefore the MFCR. Naturally, the intake axis lies on the axis of the entire domain, which is set as an axis boundary condition.

The grid independence assessment follows the approach advocated by Roche ${ }^{13}$. Three meshes were generated for Cowl1and the impact of spatial resolution on $C_{D \text { nac }}$ was evaluated under typicalcruise conditions $\left(M_{\infty}=0.85\right.$ and MFCR=0.73). The meshes had $21 \times 10^{3}, 31 \times 10^{3}$, and 
$39 \times 10^{3}$ cells, respectively. Richardson Extrapolation ${ }^{13}$ was then conducted to estimate the grid independent solution. The nacelle drag coefficient $C_{D \text { nac }}$ reduced monotonically with increasing mesh size although the difference between the medium and fine meshes was $0.2 \%$. Using a factor of safety of 1.25 , the grid convergence index $(\mathrm{GCl})$ between the medium and fine mesh solutions was $0.01 \%$ and were within the asymptotic range with a $\mathrm{GCl}$ ratio of 0.998 . The subsequent results presented in the paper are from the medium mesh solutions.

\section{Baseline airframe.}

To validate the drag computation for the airframe studies, it was vital to identify a suitable validation test case. It was decided to base the work on the NASA Common Research Model (CRM)9which is a civil transport configuration which is comparable with a typical twin-engined wide-body 250-300 seater airframe.The aerodynamics for the CRM with Through Flow Nacelles (TFN) has been experimentally investigated ${ }^{10,11,14,15}$ using a 1/37th sub-scale model at a Reynolds number of $5 \times 10^{6}$. The $\mathrm{CRM}$ has a representative modern wing design with a design point9 at $M_{\infty}=0.85$ and $C_{\mathrm{L}}$ of 0.5 . In current research the total of two Mach numbers is considered such as $M_{\infty}=0.85$ and $M_{\infty}=0.83$. A range of angles of attack from $0^{\circ}$ to $4^{\circ}$ for both Mach numbers is considered. The geometricconfigurations considered in this current research (Table 1) include theCRM Wing Body Tail with Nacelle-Pylon (WBTONP)and the sub-variants to isolate the component effects. Based on the nomenclature adopted in the original publications, the variants are Wing-Body (WB), Wing-Body with TFN and pylon (WBNP), Wing-Body with Tail (WBT0), Wing-Body-Tail with TFN and pylon (WBTONP), and pylon removed (WBTON). To complement the study the computation of isolated through-flow nacelle (TFN) was performed for the exact flow conditions as for the aircraft simulation, namely the Mach number $M_{\infty}=0.83$ at $R e=5 \times 10^{6}$ and a range of isolated nacelle incidence from $0^{\circ}$ to $6^{\circ}$.

The computations were carried out on structured grids and the gridding guidelines from the $4^{\text {th }}$ AIAA Drag Prediction Workshop (DPW4) ${ }^{16}$ were generally followed. The domain size of 
$100 c_{r e f}$ was adopted based on the conclusions of the drag workshop (Figure2) ${ }^{16}$. A medium density mesh was created ("WBTO medium") with an element count of around $10 \times 10^{6}$ elements. A grid independence assessment followed the approach advocated by Roche ${ }^{13}$. Four meshes were generated for clean wing airframe (WBT0) and the impact of spatial resolution on $C_{D}$ was evaluated under typical cruise condition $\left(M_{\infty}=0.85, A o A=2.5^{\circ}\right)$. The element refinement ratio between meshes was 1.15 in each direction. The boundary layer mesh was kept unmodified to have the same node distribution, first cell height and $y^{+}=1$ for all mesh densities. As a result, the meshes of $6.9 \times 10^{6}, 10.3 \times 10^{6}, 16.1 \times 10^{6}$, and $24.1 \times 10^{6}$ cells were generated and called "coarse", "medium", "fine", superfine" respectively (Table 1). Richardson extrapolation ${ }^{13}$ was then conductedbased on the total drag coefficient. The airframe drag coefficient $C_{D}$ reduced monotonically with increasing mesh size. Using a factor of safety of 1.25 , the second order grid convergence index $(\mathrm{GCl})$ for a medium mesh solution was $2.05 \%$ and were within the asymptotic range with a $\mathrm{GCl}$ aspect ratio of 0.985 . At the same time the second order grid convergence index $(\mathrm{GCl})$ for fine mesh solution was $1.08 \%$ and were within the asymptotic range with a $\mathrm{GCl}$ aspect ratio of 0.992 . Moreover, the convergence of residuals was observed and reached the levels of $10^{-5}$ and the convergence of total drag was monitored and the amplitude of oscillation did not exceed 1 aircraft drag count with a typical level of 0.1 drag counts.

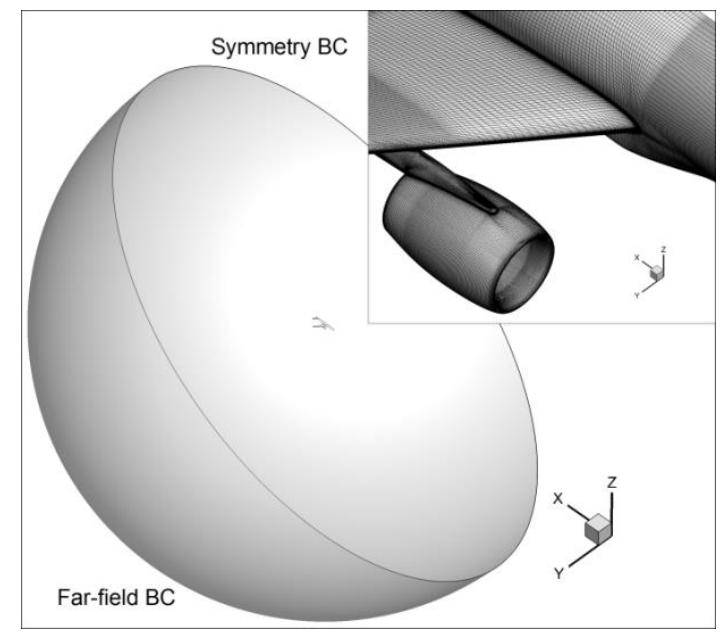

Figure2. The hemispherical domain for aircraft studies and close-up on the surface mesh of the 
nacelle

Moreover, within this work a coherent meshing guideline has been established for the creation of the mesh for the airframe with a Through Flow Nacelle (TFN). A structured mesh with a comparable blocking strategy to the clean wing case has been created (Figure2). The experience from the isolated studies was extracted in terms of required distribution of gridspacing on the nacelle surface. Those criteria have been merged with the DPW4 gridding guidelines ${ }^{16}$. Therefore the following criteria have been added: 40 elements for the nacelle lip, maximum axial spacing on the nacelle $\Delta x=L_{n a c} / 110$. Moreover, the pylon surface mesh in the longitudinal direction was dominated by the node distributions on the wing and nacelle surfaces. To complement the pylon mesh the lateral number of nodes on the trailing edge surface of the pylon was set to 13 to be compatible with the DPW4 gridding guidelines ${ }^{16}$ and the criterion for the wing trailing edge. Furthermore, the vertical node count was set to minimise the aspect ratio of surface elements and it resulted in total of 15 elements in the gulley between the nacelle and the wing. As a result,two meshes resolutions have been generated for the configuration with the throughflow nacelle (WBTONP).These meshes comprised of the existing surface mesh for the airframe parts and the introduction of a new surface mesh for the nacelle and pylon. The derivation from "WBT0 medium"surface meshreached $22 \times 10^{6}$ elements and it will be referred to as "WBTONP medium" mesh. Whereas a $30 \times 10^{6}$ element mesh was generated and denoted "WBTONP fine" mesh(Figure2), as a derivation from the "WBT0 fine" mesh.To study aerodynamic interference, it was decided to use the "WBTONP fine" mesh and to remove the pylon from the configuration to enable the impact of the pylon to be assessed.As a result a "WBTON fine" mesh was created. Furthermore a $7.2 \times 10^{6}$ million element mesh for the through-flow nacelle in isolation (Table 2) was created by following the 'fine mesh' nacelle meshing rules as the WBTONP mesh. The size of the domain for the isolated TFN computation was adopted based on the experience from isolated nacelle simulations and it is the domain radius of 50 nacelle $D_{\text {max }}$. To compare the prediction of installation effect with experiment, the "WB medium" and "WBNP medium" meshes were derived 
from previous meshes with the modification at the tail mesh only. The summary of computed mesh configuration is presented in

Table 1.

Table 1Summary of configurations and the rationale behind the computation

\begin{tabular}{|c|c|c|c|}
\hline \multicolumn{2}{|c|}{ Geometry } & $\begin{array}{l}\text { Simulation } \\
\text { type }\end{array}$ & Rationale \\
\hline$\underset{\substack{\frac{1}{4} \\
\frac{n}{3}}}{\frac{n}{3}}$ & $\begin{array}{l}\text { Cowl1 } \\
\text { Cowl3 }\end{array}$ & $\begin{array}{l}\text { 2D axissym. } \\
\text { 2D axissym. }\end{array}$ & $\begin{array}{l}\text { spillage drag and drag rise characteristics for nacelles; } \\
\text { assessment of CFD capabilities in the field }\end{array}$ \\
\hline \multirow{5}{*}{ 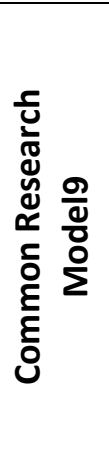 } & $\begin{array}{l}\text { CRM } \\
\text { TFN }\end{array}$ & 3D & $\begin{array}{l}\text { determine isolated nacelle drag as reference for } \\
\text { interference study }\end{array}$ \\
\hline & $\begin{array}{c}\text { WB } \\
\text { WBNP }\end{array}$ & $\begin{array}{l}3 D \\
3 D\end{array}$ & $\begin{array}{l}\text { quantify the installation effect and validate against } \\
\text { the measurements }\end{array}$ \\
\hline & WBTO & $3 D$ & $\begin{array}{l}\text { validate airframe calculations and compare against } \\
\text { the results from the AIAA DPW IV }\end{array}$ \\
\hline & WBTON & $3 \mathrm{D}$ & $\begin{array}{l}\text { Assess the interference of the airframe and the } \\
\text { nacelle }\end{array}$ \\
\hline & WBTONP & $3 D$ & Impact of pylon presence on drag characteristics \\
\hline
\end{tabular}

Table 2 The summary of mesh configurations

\begin{tabular}{c|ccccc|c} 
MESH SIZE & WB & WBNP & WBT0 & WBTONP & WBTON & TFN \\
\hline "Coarse" & & & & & \\
"Medium" & $10.2 \times 10^{6}$ & $22.4 \times 10^{6}$ & $10.3 \times 10^{6}$ & $22.4 \times 10^{6}$ & & \\
"Fine" & & & $16.1 \times 10^{6}$ & $30 \times 10^{6}$ & $30 \times 10^{6}$ & $7.2 \times 10^{6}$ \\
"Superfine" & & & $24.1 \times 10^{6}$ & & & \\
\hline
\end{tabular}

\section{Computational method}

The computations were done using animplicit flow solver (Fluent)for the entire study. The nacelle validation was carried out with use of a second order discretization scheme for both turbulent and flow terms. The Green-Gauss node based discretization was used. To achieve satisfactory 
convergence within a short time frame during the computations the Courant number (CFL) was gradually increased from 1 to 20 . The RANS turbulence modelling was applied and the SpalartAllamaras, $k-\omega S S T$, and $k-\omega S S T \gamma-\theta$ transitional models were tested.

For the aircraft studies, the second order discretization for all terms was used only initially and later substantial effort was dedicated to developing successful convergence strategy for third order computation for all terms. In this current work all results presented for the aircraft studies were done with a third order discretization scheme. Similarly, the implicit solver with Green-Gauss node based discretization was used. It has been chosen to continue with RANS turbulence modelling and, based on the results from $4^{\text {th }}$ DPW ${ }^{17}$,the $k-\omega S S T$ turbulence model was used ${ }^{18}$.

\section{Drag extraction methods}

In the experimental work on the isolated nacelles ${ }^{12}$, the spillage drag was evaluated by the wake traverse technique with use of pressure measurements taken from four rakes mounted each $90^{\circ}$ around the configuration in vertical and horizontal directions. The drag values were computed by integrating the loss momentum in the wake behind the nacelle. The major drawback of the method was the integration of relatively small velocity perturbations over an extensive area in the outer part of the experimental rake. To deal with this problem Langley et. al. ${ }^{12}$ applied a cut-off level for the head loss and it was equal $0.013 \%$. The method was tested on the numerical solution with limited agreement. For the cases of $M F C R>0.65$ and $M<0.8$, the use of a head loss criterion in conjunction with the probe spatial resolution resulted in the drag calculation being based on only 3 to 5 pitot tubes located closest to the nacelle out of 29 tubes installed in the rig.Therefore, it was needed to search for more adequate methods for the computational analyses. Moreover, the

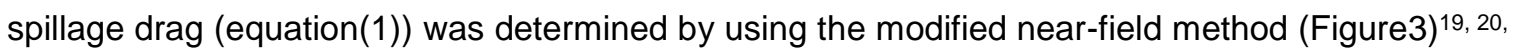
21. This method relies on the momentum integration of the pre-entry stream tubebetween the gauge stream forces $\left(F_{G 0}\right.$ and $\left.F_{G i}\right)$ at stations ' $O$ ' and 'i'which is equal to the force $F_{1}$ (equation (2)), where the individual forces $\theta_{\text {nacelle }}$ and $\Phi_{\text {nacelle }}$ are unknown. Furthermore the force $F_{2}$ (equation(3)) is known from the summation of pressure and viscous forces that act on the 
fancowl and intake diffuser from fan face (FF) to nacelle trailing edge (TE). The split of $F_{2}$ into $\theta_{\text {nacelle }}$ and $\theta_{\text {pre }}$ is dependent on the location of the stagnation point. The split between individual forces is not needed for all the cases as the drag of the nacelle is expressed by the difference between $F_{1}$ and $F_{2}$ (equation (4)), where the post-exit force is negligible at datum flow conditions of the nozzle.

$$
\begin{gathered}
C_{D \text { nac }}=\frac{F_{D}}{\frac{1}{2} \rho V^{2} A} \\
F_{1}=-\left(F_{G i}-F_{G 0}\right)=\theta_{\text {nacelle }}+\Phi_{\text {nacelle }} \\
F_{2}=\theta_{\text {nacelle }}+\theta_{\text {pre }}=\theta_{\text {nacelle }}-\Phi_{\text {pre }} \\
D_{\text {nac }}=F_{1}-F_{2}=\Phi_{\text {nacelle }}+\theta_{\text {pre }}
\end{gathered}
$$

where $D_{\text {nac }}$ is the nacelle drag, $\rho$ is the mass density of the fluid, $V$ is the velocity of the fluid and $\mathrm{A}$ is the reference area based on the maximum diameter of the nacelle. The forces that act on the inside of the streamtube are denoted as $\theta$ and the forces that act on the outside of the streamtube are denoted as $\Phi$.

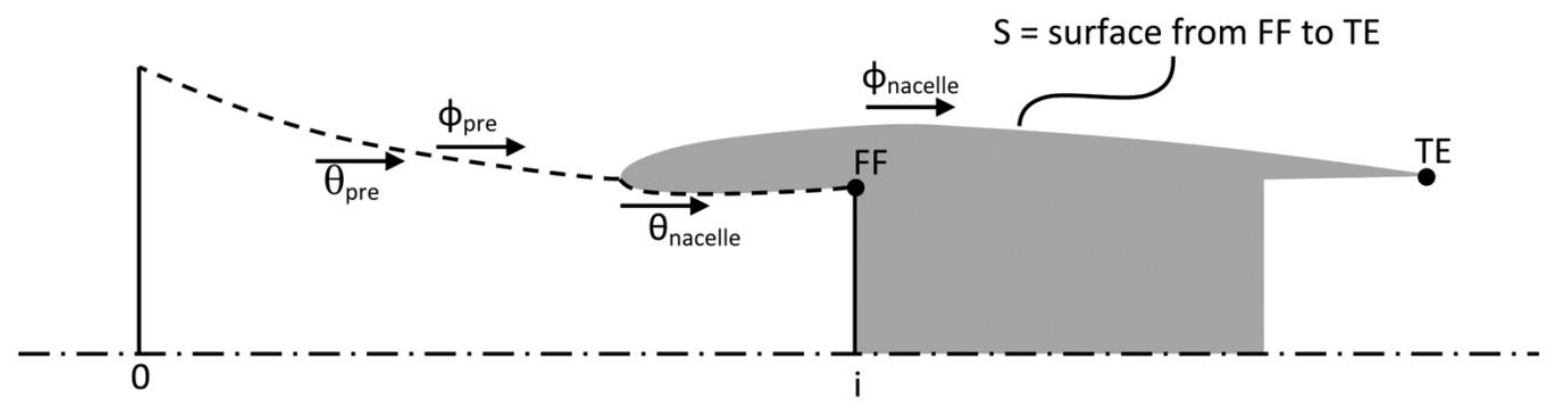

Figure3. Decomposition of modified Near Field Method forces acting on the entry streamtube and on the nacelle (based on ESDU ${ }^{19}$ )

For the aircraft cases, the entire geometry is treated as an immersed body and a standard nearfield method for force extraction is applied. Pressure and viscous forces that act on the geometry are integrated for each geometry face in the local coordinate system as normal, lateral and longitudinal to the surface. The values of drag force are grouped by components to indicate the contribution of each component like the wing, fuselage, tail, pylon and nacelle. The values of drag reported are the subject of the aerodynamic interference. To quantify the interference effect for a 
component the values of drag coefficient between different geometry variants are compared. For example, the difference between the drag values for the nacelle under the wing and the nacelle in isolation for the same flow conditions indicates the magnitude of interference drag for the nacelle due to the installation. Moreover, the proportion of viscous and pressure drag generated by selected components is reported. Furthermore, the global angle of attack is defined between the aircraft longitudinal axis and the velocity vector of the flow at upstream farfield. Finally, the projection of the vertical and axial forces from the aircraft coordinate system is done to obtain the drag and lift values for the airframe and the obtained values can be compared with experimental values from the aerodynamic balance.Even though the standard near-field method for drag extraction was chosen for a direct comparison with the experimental data, other drag extraction methods as the state-of-art far-field method ${ }^{22}$ exist. Moreover, for motorised configurations other methods have been developed such as the Modified Near Field method ${ }^{19,20,21}$ as well as the work of Malouinet $\mathrm{al}^{23}$, who proposed two new methods to compute the standard net thrust and the pre-entry thrust derived from the far-field formulation.

The numerical solutions for the aircraft studies were computed for a range of incidence from $0^{\circ}$ to $4^{\circ}$ and intervals of $0.5^{\circ}$. The experimental data ${ }^{10}, 11$ was reported at a non-uniform set of incidences up to a maximum of $10^{\circ}$. To enable the effect of the nacelle installation to be quantified, and to facilitate the comparison between the experimental and computational data sets, it was necessary to perform comparisons at constant lift so as to exclude the effect of lift induced drag. As the datasets were acquired at specified incidences, the comparisons at constant lift were enabled by performing cubic spline interpolations on the lift-drag polars.

\section{Results}

\section{Isolated nacelle studies}

Computational simulations were carried out for the range and resolution of Mach numbers from $\mathrm{M}=0.4$ to 0.9 and MFCR from 0.4 to 0.8 as used in the experiment ${ }^{12}$ (Table 3 ). To assess the drag 
rise Mach number, a range of Mach numbers from $M=0.4$ to $M=0.9$ for a single MFCR=0.7 was computed (Figure4)(Table 3). In addition, the spillage characteristics were assessed for a typical Mach number 0.85 and a range of MFCR from 0.4 to 0.73 (Figure5)(Table 3). Moreover the flow properties as in the experiment ${ }^{12}$ were recreated with Reynolds number based on nacelle maximum diameter in the range from 1.5 to 2.24 million and total pressure constant at 101,325 $\mathrm{Pa}$ and static temperature constant at $285.7 \mathrm{~K}$. The standard flow properties for dry air were used with $\gamma=1.4$ and $R=287.1 \frac{\mathrm{J}}{\mathrm{kg} \mathrm{K}}$. Furthermore, to enable clear comparisons of the experimental and computational data, as well as the differences in the sensitivities of the cowl designs to MFCR and $\mathrm{M}$, the $C_{D}$ data was normalised by the corresponding $C_{D}$ for each line at the reference conditions of MFCR=0.7 and at M of 0.7 (Figure4)and by the corresponding $C_{D}$ at the reference conditions ofMFCR=0.73 and at $\mathrm{M}$ of 0.85 (Figure5).

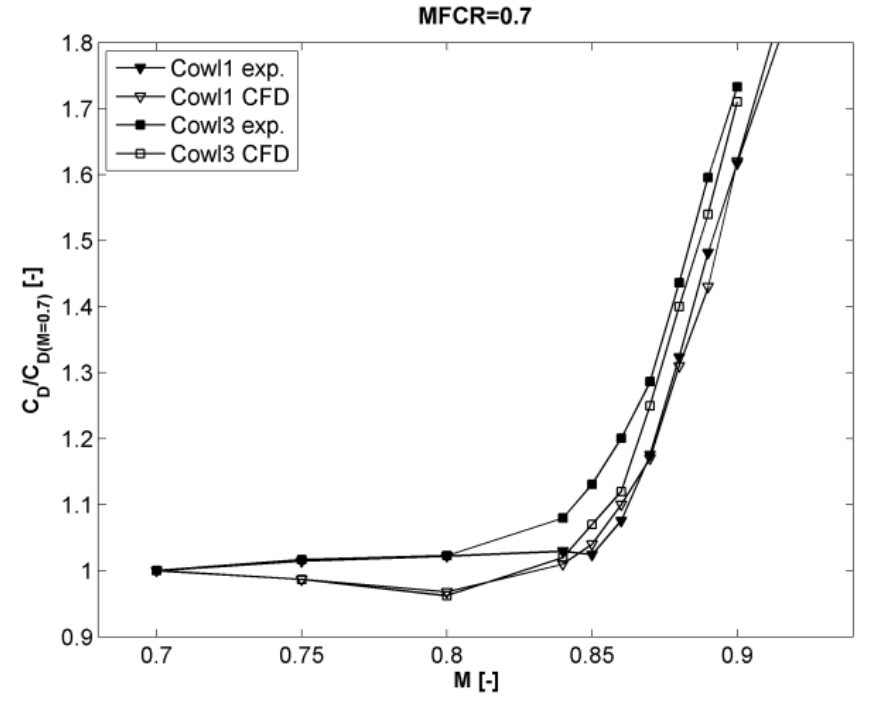

Figure4. Drag coefficient normalised by the baseline drag at $\mathrm{M}=0.7\left(C_{D} / C_{D 0}\right)$ as a function of Mach number for ARA cowls $1 \& 3$ at MFCR $=0.7 ; A o A=0^{\circ}$ compared with experimental data ${ }^{12}$.

The drag characteristics $\left(C_{D} / C_{D 0}\right)$ from the experimental results for Cowl 1 and Cowl 3 are broadly constant across the range of Mach numbers from 0.7 to 0.8 (Figure4). The insensitivity of experimental results to modest changes in drag values is explained by aninsufficient amount of Pitot probes in the measurement rig to indicate such subtle changes in the flow below the drag 
rise condition. The numerical resultsshow an initial slight decrease in $C_{D} / C_{D 0}$ as $\mathrm{M}$ increases from the 0.7 to $0.8 C_{D} / C_{D 0}$. The effect can be explained by the subtle increase of Reynolds number and an associated reduction in friction drag at conditions below drag rise. As well as determining the sensitivity of the nacelle drag to both MFCR and M, a key parameter for nacelle aerodynamic performance is the critical drag rise Mach number. It defines the Mach number at which the rise of the drag value occurs due to the change of the flow regime from subsonic to transonic. The analysis of the drag coefficient curve slope against the gradient criterion (Figure4) provides an adequate assessment of drag rise Mach number $\left(M_{D R}\right)$. The drag rise Mach number was identified as the value of the free-stream Mach number, where the gradient of drag as a function of Mach number equals $d c_{D} / d M=0.05$. This definition of drag rise Mach number was applied to both numerical and experimental datasets. A curve fit through the data points was performed and a gradient criterion was applied. With a further increase in Mach number, both cowls exhibit a typical drag rise characteristic with $M_{D R}$ of 0.84 and 0.82 , for Cowl 1 and Cowl 3, respectively. This was expected based on the design of Cowl 1 which, due to the larger leading edge radius, has a higher $M_{D R}$. The CFD shows broadly the samedrag characteristics for both cowls as compared with the experiment. TheCFD captures the relatively constant drag as the Mach number increases. Moreover, the drag rise location broadly matches the experimental results. Furthermore, in the pre-drag rise region, there is notable agreement between the CFD and measurements for both cowls with a typical difference in the order of $-15 \%$, which is explained by pressure force that acts on the aft of the nacelle due to the presence of the sting. The effect was considered in the design of the experiment andwas compensated by downstream movement of the measurement rake during calibration. Although the CFD simulations also capture the $M_{D R}$ there are very slight differences with the measurements where the calculated $M_{D R}$ are +0.005 greater than the measured value for cowl 1 and $\Delta M_{D R}=-0.007$ less for Cowl 3.

A similar parameter is considered for the sensitivity of $C_{D}$ to MFCR and is defined as $d c_{D} / d M F C R=0.05$. To analyse the accuracy of the drag coefficient computation across the 
range of Mach numbers, the calculations with constant $M=0.85$ were considered (Figure5) for a range of MFCRs from 0.4 to 0.73 (Table 3). For a constant M of 0.85 , as the MFCR is reduced the spillage drag increases for both Cowl 1 and Cowl 3 (Figure5). Cowl 1, with the larger leading edge radius of curvature, is notably less sensitive to the reduction in MFCR whereby at an extremely low MFCR of 0.40 the $C_{D} / C_{D 0}$ increased from 1.0 to 2.4. The effect is more marked for the Cowl 1 where the measured $C_{D} / C_{D 0}$ increases to 3.15 for a MFCR of 0.40 . The increase in $C_{D} / C_{D 0}$ with reducing MFCR gives rise to a critical $M F C R_{C R}$, which was numerically evaluated for the CFD results as $M F C R_{c r}=0.654$ for Cowl 1 and $M F C R_{c r}=0.58$ for Cowl 3 . In comparison with the experimental results, the CFD results indicate that the $M F C R_{c r}$ occurs at a lower MFCR and the differences between the experimental and numerical results are $\triangle M F C R_{c r}=+0.014$ and $\triangle M F C R_{c r}=+0.009$ for Cowls 1 and 3 , respectively.Overall, it was found that current numerical methods broadly captured the key nacelle drag characteristics and provided accuracy of $1.5 \%$ for the assessment of the critical MFCR and $\Delta M_{D R}= \pm 0.007$ for the drag rise Mach number. Within the context of the use of the CFD for the assessment of nacelle drag within the wider framework, these results are considered to be satisfactory.

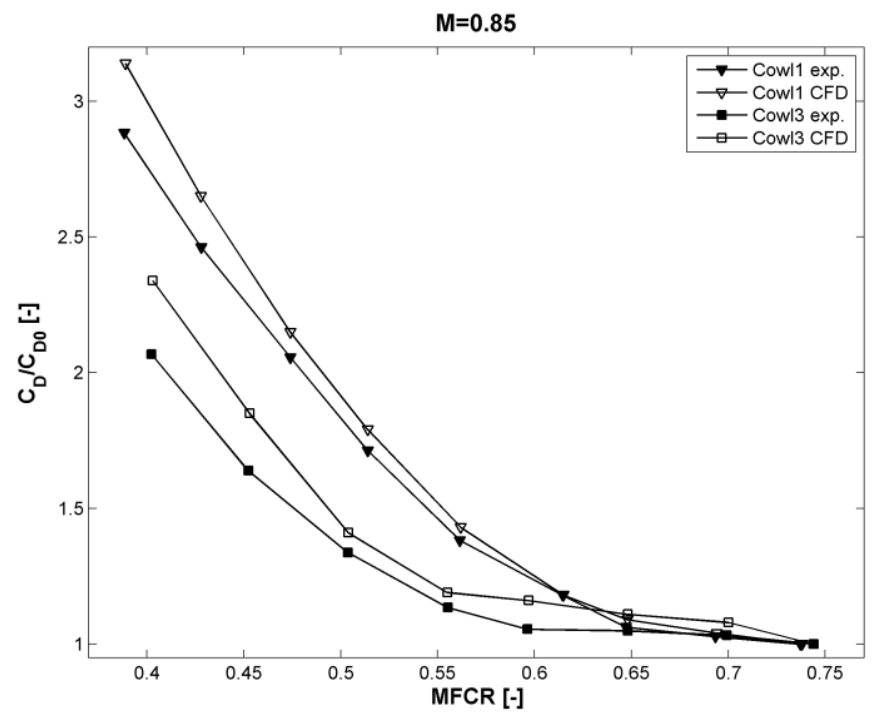

Figure5. Drag coefficient normalised by the baseline drag at MFCR $=0.73\left(C_{D} / C_{D 0}\right)$ as a function of MFCR for ARA cowls $1 \& 3$ at $R e=2.2 \times 10^{6} ; \mathrm{M}=0.85 ; A o A=0^{\circ}$ compared with experimental data ${ }^{12}$. 
Table 3 Rationale and flow configurations for ARA cowls ${ }^{12}$

\begin{tabular}{|c|c|c|c|c|c|}
\hline Geometry & $\begin{array}{c}\text { Simulation } \\
\text { type }\end{array}$ & Rationale & $A o A$ & $\boldsymbol{M}_{\infty}$ & MFCR \\
\hline \multirow{2}{*}{$\underset{\substack{\frac{\pi}{\alpha} \\
\frac{n}{3}}}{\frac{n}{3}}$} & 2D axissym. & $\begin{array}{c}\text { Assessment of drag rise } \\
\text { Mach number }\end{array}$ & $0^{\circ}$ & 0.7 to 0.9 & 0.7 \\
\hline & 2D axissym. & $\begin{array}{l}\text { Assessment of spillage } \\
\text { drag characteristics }\end{array}$ & $0^{\circ}$ & 0.85 & 0.4 to 0.73 \\
\hline
\end{tabular}

\section{Baseline aircraft studies}

Following from the foundation assessment of the CFD method for the calculation of the key drag and drag rise characteristics for an isolated nacelle, the next building block is the evaluation of the assessment of the numerical modelling for a full aircraft configuration. In particular, the extended interest lies in the generation of correlations for the assessment of engine installation drag as a function of engine size, position and operating condition. As part of this development an initial step is to evaluate the ability of CFD to determine the installation drag and change of the lift due to the installation of a through-flow nacelle.

In the current study the NASA Common Research Model9is usedas a representative vehicle of acontemporary transonic transport aircraft. The original rigid wind tunnel geometry has been used inthe current studyas the deflected geometry is not currently publically available. The issue of aerodynamic twist of the wing has been evaluated by Rivers et al. ${ }^{10}$ who reported that the twist of the wing due to the wing loading had no impact on the overall lift-drag polar. The computationswere carried out for a range of angles of attack (AoA) from $0^{\circ}$ to $4^{\circ}$ in half a degree steps. The experimental setup was recreated ${ }^{10}$ for $R e=5 \times 10^{6}$ and Mach $=0.85$ with a static temperature $T_{s t}=322 \mathrm{~K}$ and the following constants $R=287.1 \frac{\mathrm{J}}{\mathrm{kg} \mathrm{K}}, \gamma=1.4$. The summary of computed flow conditions for each geometry configuration is listed in Table4. Furthermore, the mesh sensitivity study was computed with fully turbulent $k-\omega S S T$ modeland compared with experimental data from Rivers et al. ${ }^{10}$ (Figure 6). In the experiment a set of trip dots was used to 
fix the location of transition to allow for comparison with CFD. The trip dots were applied on the fuselage, on the nacelle, at $10 \%$ of wing chord for both sides of the wing and at $10 \%$ of tail chord on both sides of the tail ${ }^{10}$. The results were also compared(Figure 6). with the range of benchmarkfully turbulent solutions from the ${ }^{\text {th }}$ Drag Prediction Workshop (DPW) ${ }^{17}$.

In the current study, two mesh resolutions are used for the aircraft study. A mesh resolution of $10 \times 10^{6}$ elements is referred to as a "medium" mesh and the mesh resolution of $16 \times 10^{6}$ elements is referred to as "fine" mesh. At condition of $R e=5 \times 10^{6}$ and $M_{\infty}=0.85$, the medium mesh provides results comparable to a wide range of results from the $4^{\text {th }}$ DPW as reported by Tinoco ${ }^{17}$ (Figure 6). However, the computed drag is over-predicted by approximately $\Delta C_{D}=+27 d c$ for a range of lift coefficients $C_{L}$ from 0.1 to 0.4 . With a further increase of $C_{L}$, the results for the medium mesh reduced the discrepancy to $\Delta C_{D}=+20 \mathrm{dc}$ at $C_{L}=0.5$. Moreover, the results for the fine mesh improved the accuracy in comparison to the medium mesh (Figure 6). At the design point $C_{L}=0.5$ the drag over-prediction is $\Delta C_{D}=+13 d c$. Although the fine mesh results are wellaligned with the experimental drag polar above $C_{L}>0.5$, some discrepancy at lower values of $C_{L}$ is observed. The discrepancy for a range of $C_{L}$ from 0.1 to 0.4 is around $\Delta C_{D}=+21 d c$. The summary of agreement was provided in Table 5.

Table4 Flow conditions for the computed configurations

\begin{tabular}{|c|c|c|c|c|c|}
\hline \multirow[t]{2}{*}{ Geometry } & & \multicolumn{4}{|c|}{ Flow conditions } \\
\hline & & $\mathbf{R e}$ & $\mathbf{M}$ & MFCR & AoA \\
\hline \multirow{6}{*}{ 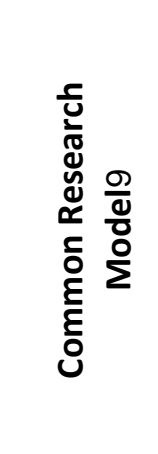 } & CRM TFN & $5 \times 10^{\wedge} 6$ & 0.83 & $\begin{array}{l}M F C R \\
\approx 0.66\end{array}$ & $0^{\circ}$ to $6^{\circ}$ \\
\hline & WB & $5 \times 10^{\wedge} 6$ & 0.83 and 0.85 & $\mathrm{~N} / \mathrm{A}$ & $0^{\circ}$ to $4^{\circ}$ \\
\hline & WBNP & $5 \times 10^{\wedge} 6$ & 0.83 and 0.85 & $\begin{array}{l}M F C R \\
\approx 0.66\end{array}$ & $0^{\circ}$ to $4^{\circ}$ \\
\hline & WBTO & $5 \times 10^{\wedge} 6$ & 0.83 and 0.85 & N/A & $0^{\circ}$ to $4^{\circ}$ \\
\hline & WBTON & $5 \times 10^{\wedge} 6$ & 0.83 & $\begin{array}{l}M F C R \\
\approx 0.66\end{array}$ & $0^{\circ}$ to $4^{\circ}$ \\
\hline & WBTONP & $5 \times 10^{\wedge} 6$ & 0.83 & $\begin{array}{l}M F C R \\
\approx 0.66\end{array}$ & $0^{\circ}$ to $4^{\circ}$ \\
\hline
\end{tabular}




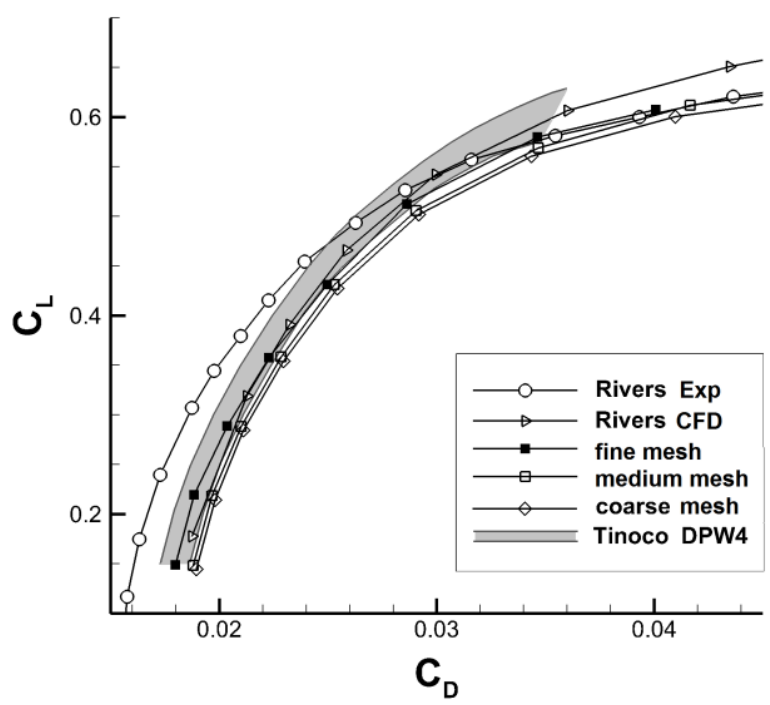

Figure 6. Computational drag polar from for clean wing CRM (WBT0) for 'coarse, 'medium and 'fine mesh' compared with computational data for rigid geometry by Rivers et al and measurements ${ }^{10}$ using $k-\omega S S T$ and S-A models; band of structured-mesh solutions from 4th Drag Prediction Workshop marked in grey ${ }^{17}$; all data at $R e=5 x 10^{6}$ and $M_{\infty}=0.85$; all current CFD with $k-\omega S S T$.

To better understand the results, a series of computations for three different turbulence models was carried out (Figure7). The tested models were the Spallart-Allmaras, k- $\omega$ SST and k- $\omega$ SST $\mathrm{Y}-\theta$ transitional model. All three models were examined using the medium mesh. The transitional SST model presented notable agreement for $C_{L}=0.5$ with $\Delta C_{D}=+6 d c$. Moreover, a relatively low discrepancy of $\Delta C_{D}=+13 d c$ is observed between thek- $\omega$ SST $\mathrm{\gamma}-\theta$ transition model and the measurements for a range of $C_{L}$ from 0.1 to 0.4 . The quoted discrepancy of $\Delta C_{D}=+13 d c$ is significantly better as compared with the computationalresults for k- $\omega$ SST and the discrepancy of $\Delta C_{D}=+27 d c$ in the same range of $C_{L}$. However, it has to be noted that the transition in the model was not forcedto match the experiment arrangement. As a result, the transitional $k-\omega$ SST $y-\theta$ under-predicts drag above $C_{L}=0.55$. Therefore, modelling of the transition needs to be treated with caution and in this study, to avoid any spurious effects due to the uncontrolled transition modelling, the standard k- $\omega$ SST turbulence model has been used throughout. Within the current study, the Spallart-Allmaras model preformed relatively badly and thus wasnotconsidered in 
further analyses.Overall, a k-w SST model has been chosen as the baseline turbulence model for the current study, as it is consistent with the previous Drag Prediction Workshop computational activities.Moreover, the k- $\omega$ SST turbulence model has been used throughout the study to avoid any spurious effects due to the uncontrolled transition modelling. It was decided not to use the fine tuning of the transition model for individual cases and this was not considered sufficiently robust.Also the good knowledge of the k-w SST model allows to use the most aggressive convergence strategy and to critically assess the results.

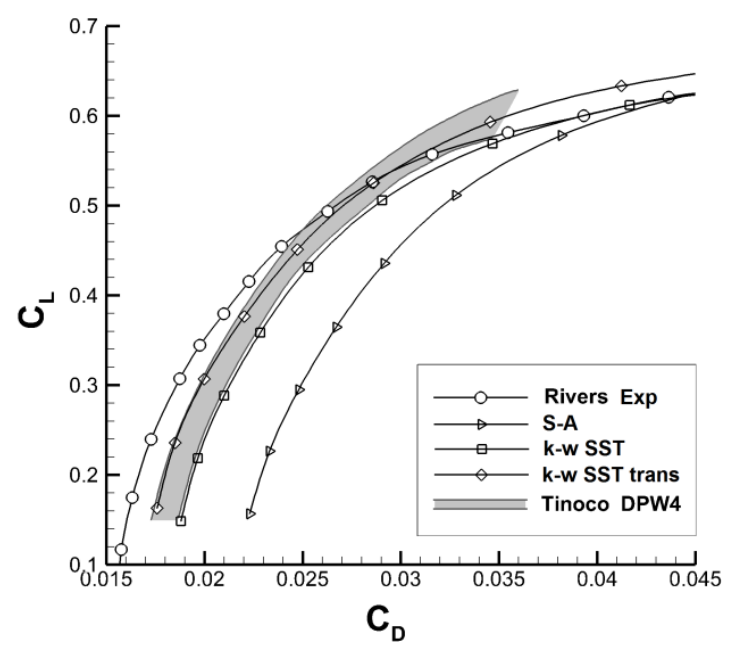

Figure7. Computational drag polar from for rigid clean wing CRM (WBT0) for 'medium mesh' for different turbulence models: Spalart-Allmaras (S-A) and k- $\omega$ SST and transitional k- $\omega$ SST; all data compared with measurements ${ }^{10}$; all data at $\operatorname{Re}=5 \times 10^{6}$ and $M_{\infty}=0.85$;

As well as considering the overall drag polar for the CRM, the computed wing surface pressure distributions were also compared with the measured data at selected stations (Figure 8) for the aircraft design point of $\mathrm{C}_{\mathrm{L}} \approx 0.5$ and $\mathrm{M}=0.85$ (Figure9). It was chosen to compare the pressure distribution at an almost constant value of lift coefficient. Therefore, the experimental data for $\mathrm{C}_{\mathrm{L}}=0.494$ and corresponding $\mathrm{AoA}=2.90^{\circ}$ was compared with the numerical data at $\mathrm{C}_{\mathrm{L}}=$ 0.51 and $\mathrm{AoA}=2.50^{\circ}$, which is the closest comparison available. A cross-section of the wing at wingspan position of $y / b=\eta=0.283$ was chosen (Figure 8 ), due to its greatest relevance to 
installation studies for through flow nacelles. Although good agreement is observed, the peak value of pressure distribution is slightly under-predicted by $\Delta C_{p}=-0.04$ for the medium mesh (Figure9). Moreover, the medium mesh computed the shock location $\Delta x / c=+0.05$ downstream from its experimental location. At the same time the medium mesh accurately evaluated thepressure coefficient at the shock location of $C_{p}=-0.67$. For the same flow conditions, but with use ofthe fine mesh (Figure9), the peak value of pressure coefficient was under-predicted by $\Delta C_{p}=-0.04$. However, the shock position was computed accurately at $x / c=0.55$ and the pressure coefficient was over-predicted by $\Delta C_{p}=+0.08$. Overall the CFD results show good agreement with the key aspects of the measured data such as peak $\mathrm{Cp}$ and shock location. There is a small improvement in the agreement when a finer mesh is used, although overall the differences are modest.It can be concluded that 'medium mesh' is sufficient within the current scope. Unless otherwise stated, the results from the medium mesh simulations are used in this paper.

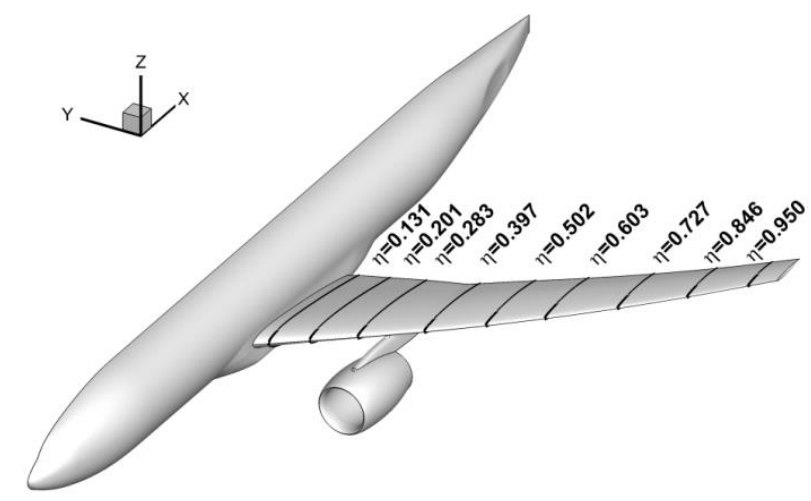

Figure 8. Wing cross-sections with experimental datasets and their location on CRM WBNP 


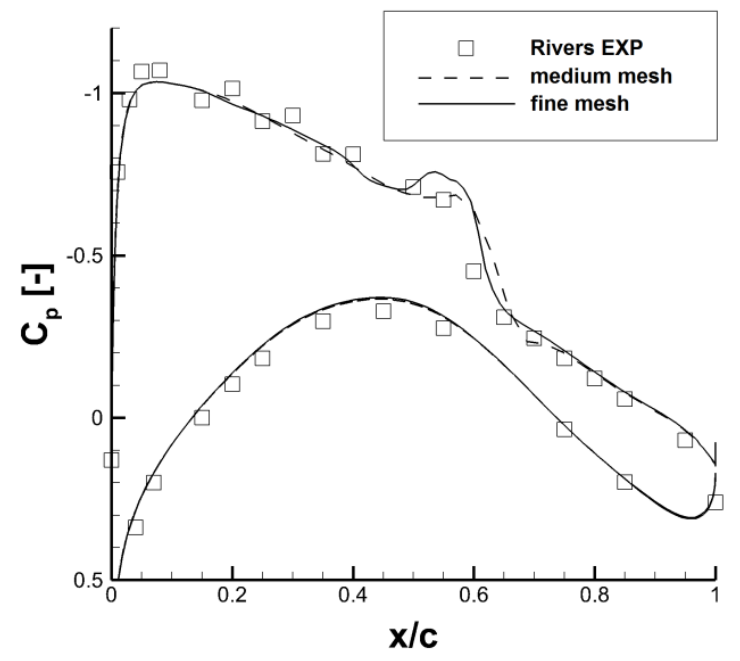

Figure9.Pressure distribution $c_{p}$ for clean wing CRM (WBT0) for two mesh resolutions 'medium' and 'fine' at $A o A=2.5^{\circ} ; C_{L}=0.51 ; \eta=0.283$; both with structured grid and k- $\omega$ SST turbulence model; numerical results compared with experiments by Rivers et al ${ }^{10}$ at $A o A=2.90^{\circ} ; C_{L}=0.494 ; \eta=$ 0.283 ; All data at $R e=5 \times 10^{6}$ and $M_{\infty}=0.85$.

\section{Effect of Through Flow Nacelle (TFN)}

To investigate the impact of a through flow nacelle (TFN) on the overall aircraft aerodynamics, the simulations for Wing-Body (WB) and Wing-Body-Nacelle-Pylon (WBNP)(Figure 8)configurations were computed and compared with the experimental dataset at Mach number 0.83 for a range of angles of attack from $0^{\circ}$ to $4^{\circ}$. The placement of a TFN on the airframe has a notable effect on the airframe aerodynamics. The measured drag coefficient increased by approximately $\Delta C_{D}=+25 d c$ for lift coefficients in the range of $C\llcorner$ from 0.15 to 0.4 at Mach 0.83 (Figure 10). The nominal cruise design lift coefficient is 0.5 and from a $C_{L}$ of 0.4 to 0.65 the increase in aircraft drag coefficient due to the installation of the TFN gradually reduces from approximately $25 \mathrm{dc}$ to a value of zero at $C_{L}=0.65$ (Figure 10). As $C_{L}$ increases further beyond 0.65 , the effect of the TFN is to reduce the overall $C_{D}$. The agreement between the numerical data and the measurements is as good as for the clean wing configuration (Table 5). Although there is an off-set in drag levels of approximately $10 \mathrm{dc}$ between the fully turbulent computation and the experimental data (Figure 10), the CFD results also capture these key elements of the impact of the TFN on the overall aircraft $C_{L}$ and $C_{D}$ (Figure 10). For example, the CFD shows a typical increase in $C_{D}$ of 
approximately $+25 \mathrm{dc}$ at relatively low lift coefficients of $C_{L}=0.15$ and the calculated point at which the change in $C_{D}$ reduces to zero is $C_{L}=0.60$ in comparison with $C_{L}=0.65$ for the experimental data. In addition, the CFD similarly shows that at greater values of $C_{L}$, there is an overall reduction in $C_{D}$ due to the presence of the TFN. The use of $k-\omega S S T \gamma-\theta$ improved the accuracy and resulted in typical offset of $2 \mathrm{dc}$ between the computation and the measurements. The impact of the TFN on $C_{D}$ can be considered in more detail by evaluating the differences between the clean wing configuration (WB) and the TFN configuration (WBNP) at a constant lift coefficient (equation(5) andFigure11).

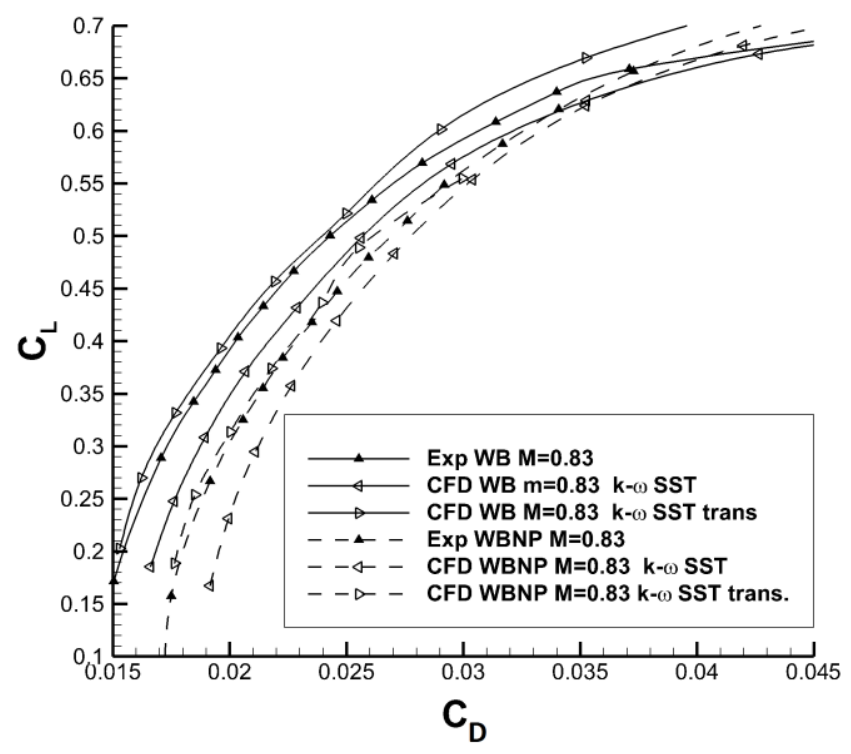

Figure 10. Lift-drag polar for CRM with (WBNP) and without a TFN (WB) for "Medium" mesh; computed with $k-\omega S S T$ and $k-\omega S S T \gamma-\theta$ transitional model; numerical results compared with experimental by Rivers et $\mathrm{al}^{10}$ at $R e=5 \times 10^{6} ; M=0.83$.

The impact of the TFN on the overall aircraft drag shows that the magnitude of the effect of installation drag notably depends on the aircraft $C_{L}$ (Figure11). At M 0.83 and with use of $k-$ $\omega S S T$ turbulence model the $\Delta C_{\text {Dinst }}$ is relatively constant at $25 \mathrm{dc}$ up to a $C_{L}$ of 0.5 , beyond which it then reduces rapidly and eventually becomes a beneficial change of $\Delta C_{\text {Dinst }}=-0.020$ at $C_{L}=$ 
0.675. The results of transitional model $k-\omega S S T \gamma-\theta$ offer worse agreement than fully turbulent calculation as an opposite trend to the experimental data is observed for $C_{L}>0.5$. It was understood that a free-transition model was used in the experiment and thus the transition occured at different $\mathrm{x} / \mathrm{c}$ location for configurations with the TFN as compared to the clean-wing configuration. The fully turbulent computation at $\mathrm{M}=0.85$ is broadly similar to the results of $k-$ $\omega S S T$ computation for $\mathrm{M}=0.83$ although the $C_{L}$ at which the $\Delta C_{\text {Dinst }}$ begins to reduce is slightly lower. In addition to the effect on the drag, the presence of the TFN affects the lift coefficient. The effect on lift coeffcient $\left(C_{L}\right)$ is evaluated by considering the differences at a constant angle of attack (AoA) (equation (6)). The impact on $C_{L}$ as a function of AoA shows a detrimental loss of lift across the range of AoA from 0 to about 3 (Figure12). As the AoA increases beyond $3^{\circ}$, there is a beneficial increase in $\Delta C_{L}$ to about +0.02 . Overall this indicates a change in lift coefficient from a loss of about $15 \%$ at $A o A=0^{\circ}$ to an increase of lift coefficient of about $3 \%$ at AoA of $4.5^{\circ}$. The sensitivity of $\Delta C_{L}$ to Mach number is relatively modest.

$$
\begin{aligned}
& \Delta C_{\text {Dinst }}\left(C_{L}\right)=\left[C_{D(W B N P)}-C_{D(W B)}\right]_{C_{L}=\text { const }} \\
& \Delta C_{L \text { inst }}(A o A)=\left[C_{L(W B N P)}-C_{L(W B)}\right]_{A o A=\text { const }}
\end{aligned}
$$

Overall, although the CFD captures the main elements of the measured installation effects, there are also some differences. The comparison at constant lift between the datapoints for both experimental and numerical data was performed. As a result at $\mathrm{M}=0.83$ and low $C_{L}$ values the CFD shows good agreement with the measured $\Delta C_{\text {Dinst }}$ of $25 \mathrm{dc}$ (Figure11). The CFD also shows a reduction in $\Delta C_{\text {Dinst }}$ with an increase in $C_{L}$. At $\mathrm{M}=0.85$, the agreement between the CFD and the measurements is not as good across the low $C_{L}$ range, although the reduction of $\Delta C_{\text {Dinst }}$ at higher $C_{L}$ is better with very good agreement at a high $C_{L}$ of 0.5 (Figure11). The $\Delta C_{L}$ as a function of AoA (Figure12) are generally captured well by the CFD with the characteristic constant $\Delta C_{\text {Linst }}$ at lower AoA followed by an increase at an AoA of approximately $3^{\circ}$ for both $M=0.83$ and $M=0.85$. 


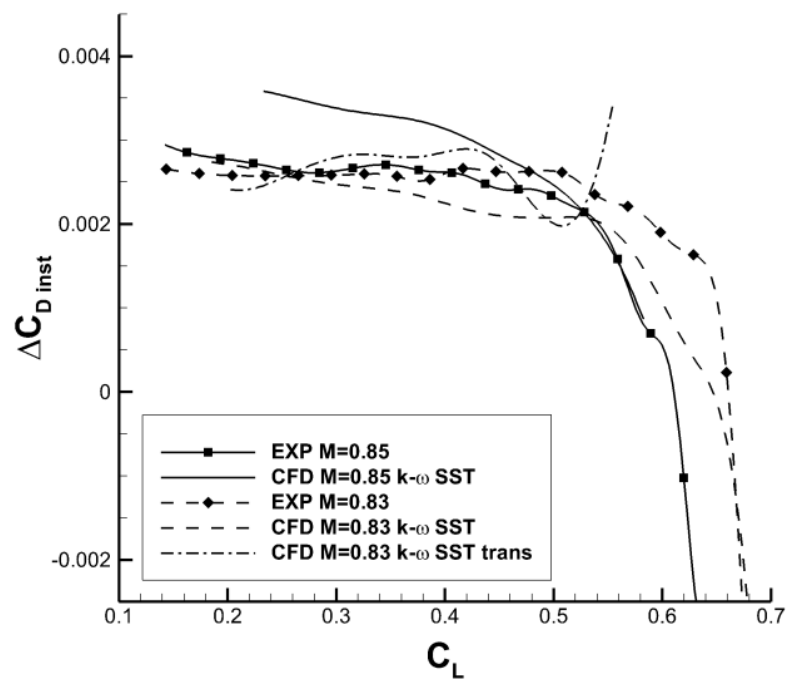

Figure11. Installation drag coefficient ( $\Delta C_{\text {Dinst }}$ ) for CRM WB and WBNP subscale as function of lift coefficient; comparison between CFD medium mesh and experimental data by Rivers et al ${ }^{10}$ for Mach number of 0.85

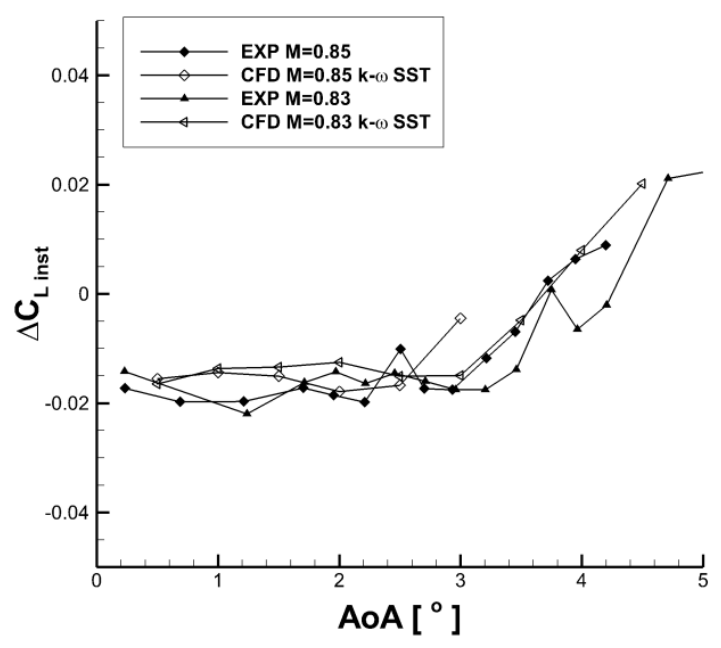

Figure12. Installation lift coefficient ( $\Delta C_{\text {Linst }}$ ) for CRM WB and WBNP subscale as function of lift coefficient; comparison between CFD medium mesh and experimental data for Mach number of 0.85

Clearly the TFN can have a notable impact on the overall changes in $C_{D}$ and $C_{L}$. It is also of interest to consider how the TFN affects the more local aerodynamics of the wing. The presence of the TFN changes the pressure coefficient distribution mostly at measurement stations closest to the installation such as spanwise positions of $\eta=0.201, \eta=0.283$ and $\eta=0.397$ (Figure 8). 
As a datum, it was chosen to present the $C_{p}$ distributions for the computations and the measurements for the clean wing (WB) configurations at $\mathrm{M}=0.83$ and equal lift of $C_{L}=0.5$ (Figure 13). Based on the measurements, the installation effect between the configurations with (WBNP) and without the nacelle (WB) was presented at a constant angle of attack of $A o A_{E X P}=$ $2.87^{\circ}$ (Figure 13). The purpose of this comparison is to indicate the loss of lift and the change in the pressure distribution due to the installation.Furthermore, the $C_{p}$ distributions for the computations of WBNP configuration were considered (Figure 13). Ideally, the comparison between the computations and measurements for WBNP would be done at a constant lift coefficient, but the closest available data was for $\left(C_{L}\right)_{W B N P C F D}=0.483$ and $\left(C_{L}\right)_{W B N P E X P}=0.479$, respectively. Although there is a difference of $\Delta C_{L}=0.004$, it is estimated that this equates to a difference in maximum $\Delta C_{p}$ of 0.0047 . This is significantly smaller that the $\Delta C_{p}=0.1$ due to the effect of the TFN installation.

Based on the experimental results, the inboard section of the nacelle $(\eta=y / b=0.201$; Figure 13) is subject to an increased local flow acceleration on the suction side relative to the clean wing. The increased suction starts at $\mathrm{x} / \mathrm{c}=0.1$ and can be quantified as an increase of $\Delta C_{p}=0.1$, which increases the local peak isentropic Mach number from 1.42 to 1.46 . The constant offset of $\Delta C_{p}=0.1$ between the clean wing and TFN configuration holds until the shock region. Further outboard (Figure14), at the cross-section closest to the engine installation $(\eta=0.283)$, the relative effects on the pressure distribution diminished. The key difference is that the position of the shock moved forward from $\mathrm{x} / \mathrm{c}=0.45$ for the clean wing to $\mathrm{x} / \mathrm{c}=0.35$ with TFN for the experimental data. There is evidence of the impact of the TFN on the pressure side (Figure14), where there is a reduction of the pressure coefficient inboard from the pylon, followed by an increase of pressure coefficient after the nacelle trailing edge at $x / c=0.15$. The change of pressure coefficient is approximately $\Delta C_{p}= \pm 0.1$. Further aft on the pressure side at $\mathrm{x} / \mathrm{c}=0.7$ the pressure distribution for both configurations (WBT0 and WBTONP) are broadly unaffected. 


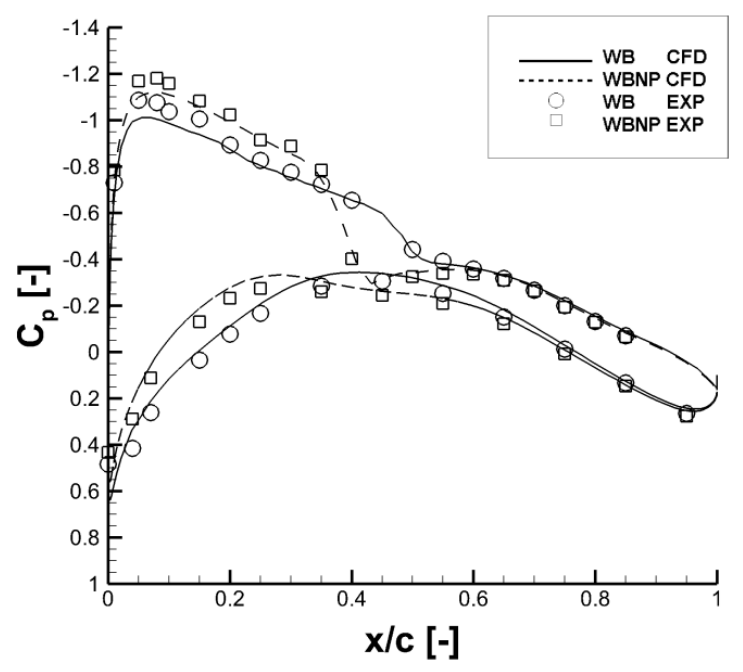

Figure 13. Comparison of pressure distribution $C_{p}$ for CRM with (WBNP) and without the TFN (WB) at $R e=5 \times 10^{6} ; M=0.83 ; \eta=0.201$; computation with $k-\omega S S T$ fully turbulent; clean wing (WB) $C_{L}=$ 0.5 for both experimental and numerical; experimental comparison between WB and WBNP at constant angle of attack $A o A_{E X P}=2.87^{\circ}$; numerical comparison between WB and WBNP at constant angle of attack $A o A_{E X P}=2.5^{\circ}$.

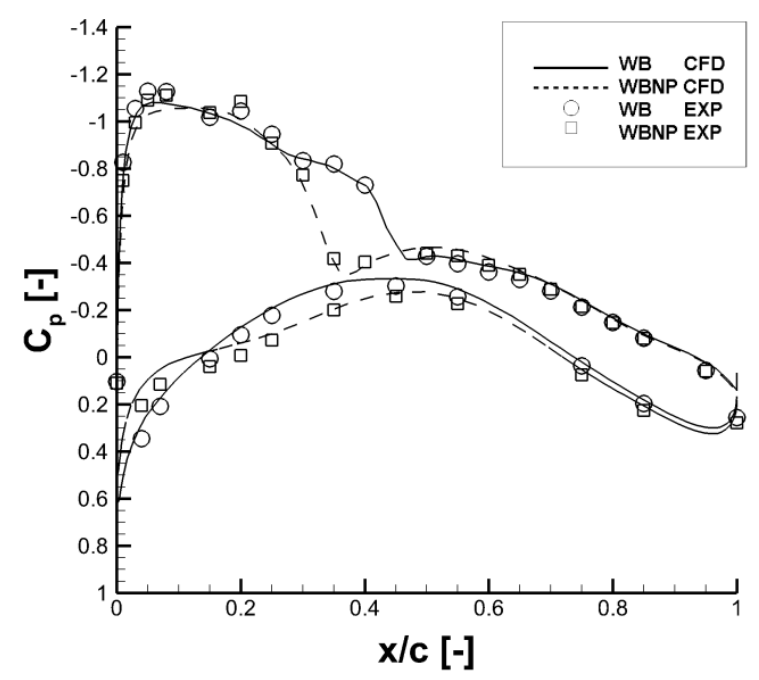

Figure14. Comparison of pressure distribution $C_{p}$ for CRM with (WBNP) and without the TFN (WB) at $R e=5 \times 10^{6} ; M=0.83 ; \eta=0.283$; Computation with $k-\omega S S T$ fully turbulent; clean wing (WB) $C_{L}=$ 0.5 for both experimental and numerical; experimental comparison between WB and WBNP at constant angle of attack $A o A_{E X P}=2.87^{\circ}$; numerical comparison between WB and WBNP at constant angle of attack $A o A_{E X P}=2.5^{\circ}$. 
Although the computations tend to under-predict the suction peak value of pressure coefficient by $\Delta C_{p}=-0.1$ (Figure 13 and Figure14), the under-prediction is consistent between the clean wing configuration and configuration with the TFN. Moreover, the location of the shock is captured correctly for the configurations with and without the nacelle. As well as for the suction side, the computation for the pressure side of the wing is in a good agreement with measurements. The CFD captured correctly the magnitude of flow acceleration in the gulley between the wing and the nacelle with maximum $\Delta C_{p}=+0.15$ at $\mathrm{x} / \mathrm{c}=0.15$ (Figure 13). Even though, in case of some crosssections the experimental readings were not complete $(\eta=0.397$, Figure15), it can be judged based on the agreement with the available experimental data (Figure15) thatthe results further outboard at $\eta=0.397$ are found to be credible where comparisons can be made(Figure15). The presence of the installation is found to shift the shock location by $\Delta x / c=-0.1$ and causes a reduction is peak pressure coefficient of $\Delta C_{p}=-0.2$.

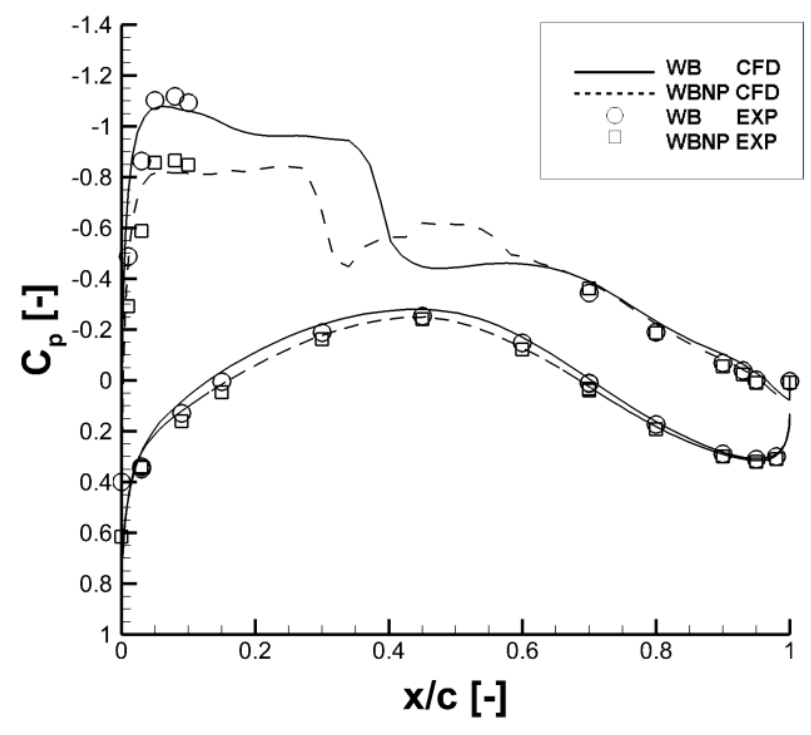

Figure 15. Comparison of pressure distribution $C_{p}$ for CRM with (WBNP) and without the TFN (WB) at $R e=5 \times 10^{6} ; M=0.83 ; \eta=0.397$; Computation with $k-\omega S S T$ fully turbulent; clean wing (WB) $C_{L}=$ 0.5 for both experimental and numerical; experimental comparison between WB and WBNP at constant angle of attack $A o A_{E X P}=2.87^{\circ}$; numerical comparison between WB and WBNP at constant angle of attack $A o A_{E X P}=2.5^{\circ}$. 
The overall changes to the pressure distribution link to the loss of total lift for the TFN configuration in relation to clean wing. The change comes from the loss of negative $C_{p}$ on the suction side of the wing as well as stronger shock interaction. Overall, the CFD results indicate similar characteristics to the experimental data.

Table 5Differences between experimental and numerical values of drag coefficient in drag counts (dc) for chosen configurations

\begin{tabular}{|c|c|c|c|c|c|c|c|c|c|c|}
\hline Geometry & \multicolumn{4}{|c|}{ WВTO } & \multicolumn{3}{|c|}{ WB } & \multicolumn{3}{|c|}{ WBNP } \\
\hline $\boldsymbol{M}_{\infty}$ & 0.85 & 0.85 & 0.85 & 0.85 & 0.85 & 0.83 & 0.83 & 0.85 & 0.83 & 0.83 \\
\hline Mesh & med & fine & med & med & med & med & med & med & med & med \\
\hline Turbulence & $\begin{array}{l}k-\omega \\
\text { SST }\end{array}$ & $\begin{array}{l}k-\omega \\
\text { SST }\end{array}$ & $\begin{array}{c}\mathrm{k}-\omega \mathrm{SST} \\
\text { trans }\end{array}$ & S-A & $\begin{array}{l}k-\omega \\
\text { SST }\end{array}$ & $\begin{array}{l}k-\omega \\
\text { SST }\end{array}$ & $\begin{array}{c}\mathrm{k}-\omega \mathrm{SST} \\
\text { trans }\end{array}$ & $\begin{array}{l}k-\omega \\
\text { SST }\end{array}$ & $\begin{array}{l}k-\omega \\
\text { SST }\end{array}$ & $\begin{array}{c}\mathrm{k}-\omega \mathrm{SST} \\
\text { trans }\end{array}$ \\
\hline$C L=0.3$ & 27 & 21 & 13 & 64 & 12 & 14 & -4 & 18 & 13 & -1 \\
\hline$C L=0.4$ & 26 & 22 & 12 & 53 & 13 & 14 & -4 & 17 & 13 & -1 \\
\hline$C L=0.5$ & 20 & 13 & 6 & 55 & 12 & 14 & -5 & 13 & 9 & -8 \\
\hline
\end{tabular}

\section{Drag decomposition}

To examine the decomposition of the drag and itssensitivity to the angle of attack, the WBTONP configuration using the fine mesh was simulatedat $M=0.83$ for a range of $A \circ A$. $A$ drag breakdown into pressure and viscous forces was initially analysed. The interest in the specific pressure and viscous contributions is driven by the desire to develop reduced order methods for preliminary design assessments. The contribution of pressure and viscous forces to the drag was analysed as a proportion of total drag of an aircraft at each operating point (Figure 16). The total pressure contribution for all components varies from $40 \%$ at zero incidence up to $60 \%$ at incidence of $A o A=3^{\circ}$. The absolute levels of the viscous contribution broadly remained constant with angle of attack for each of the individual components(Figure 16b). As a result, the relative viscous contribution drops as the pressure field becomes dominant at higher angles of attack. It is noted that at $A o A=1^{\circ}$ the contribution of pressure drag on the nacelle is of the same order of 
magnitude as the contribution of the fuselage (Figure 16a), even though the nacelle is a far smaller entity.

a)

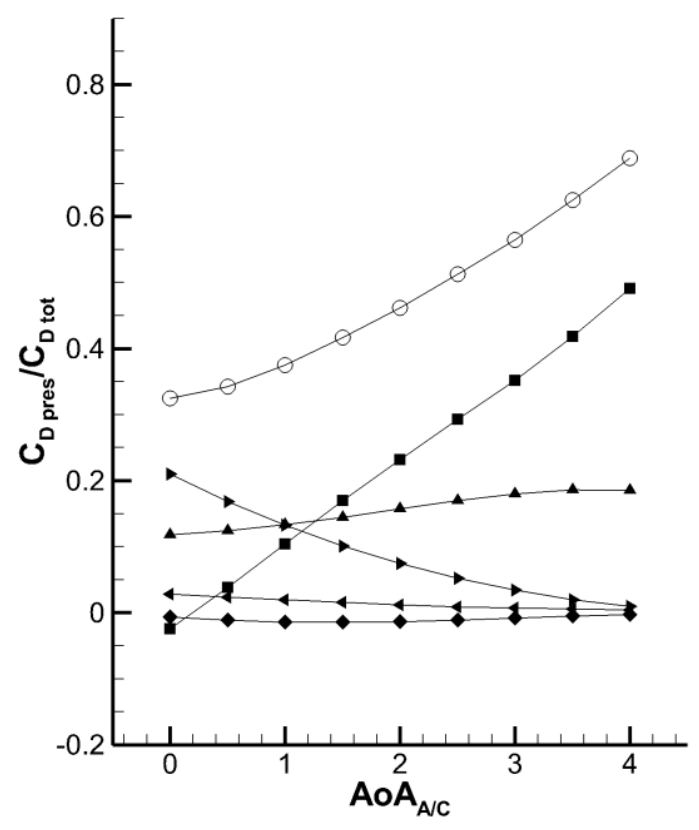

b)

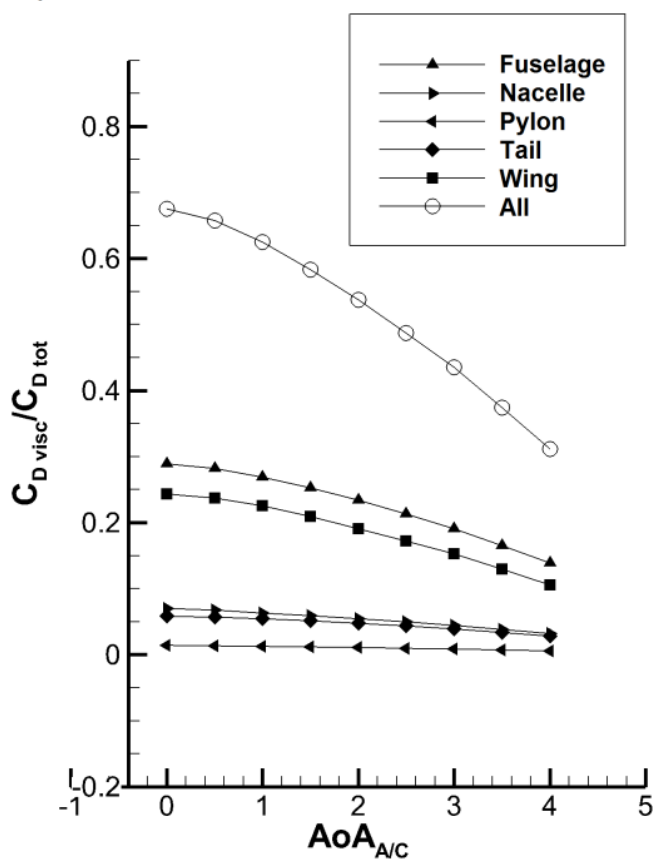

Figure 16. (a) pressure and (b) viscous contributions to total drag coefficient for CRM WBTONP subscale as function of aircraft angle of attack; results for CFD fine mesh data for Mach number of 0.83

Furthermore, to observe the characteristics of the drag coefficient for the nacelles and pylons in greater detail a comparison is presented for selected aircraft components only (Figure17). Of particular interest is the contribution of the pylon as well as the difference in the nacelle drag between an installed TFN and an isolated uninstalled TFN of the CRM. To allow a comparison with the isolated nacelle, additional 3D simulations of the isolated through-flow nacelle were carried out and the incidence of the nacelle was varied to cover the entire range of interest. The drag coefficient for two isolated nacelles is presented in aircraft drag counts to be comparable with other data computed in the presence of the airframe.Based on the WBTONP configuration, the value of the drag coefficient computed with use of standard near-field method at $A \circ A=0^{\circ}$ for the nacelle on the airframeis $C_{D \text { nac }}=60 d c$ and for the pylonis $C_{D \text { pyl }}=9 d c$. The drag values presented are the pressure and forces integration on the surfaces of the nacelle and the pylon 
and they are subjected to aerodynamic interference. As for the pylon, the contribution (Figure17) is relatively insensitive to $A \circ A$ and reduces from $9 \mathrm{dc}$ to $5 \mathrm{dc}$ for two pylons in the range of $\mathrm{AoA}$ from $0^{\circ}$ to $4^{\circ}$.As for the nacelle,the nacelle drag component drops down with an increase of aircraft angle of attacksignificantly from 60 aircraft drag counts (dc) at $A o A=0^{\circ}$ down to $20 \mathrm{dc}$ at $A o A=4^{\circ}$. This observable drop is explained by the effect of the pressure field from the underside of the wing which acts on the nacelle. As the wing loading increases with AoA, there is an increase in the pressure on the aft of the nacelle which reduces the pressure $\operatorname{drag} C_{D \text { pres }}$ and counteracts the drag contribution from the rest of the nacelle and which dominatesat zero incidence. To evaluate aerodynamic interference, the configuration without a pylon (WBTON) was computed. The net result betweenconfigurations with (WBTONP) and without the pylon (WBTON)was computed and comprises of the pylon drag, the mutual interference drag between the pylon and the wing, and the mutual interference drag between the pylon and the nacelle. This net difference reduces monotonically from $+9 \mathrm{dc}$ at $A o A=0^{\circ}$ to $+1 \mathrm{dc}$ at $A o A=4^{\circ}$. As for the nacelle, the computation of WBTON resulted in broadly the same trend for nacelle drag coefficient (Figure17) as the WBTONP configuration. Moreover, the value of $C_{D n a c}$ at a cruise $\mathrm{AoA}=2.5^{\circ}$ is the same between the configurations with and without the pylon. The largest differences between the computations are observed for a range of incidence from $A o A=0^{\circ}$ to $A o A=1.5^{\circ}$, however the magnitude of difference is less than $6 \mathrm{dc}$. 


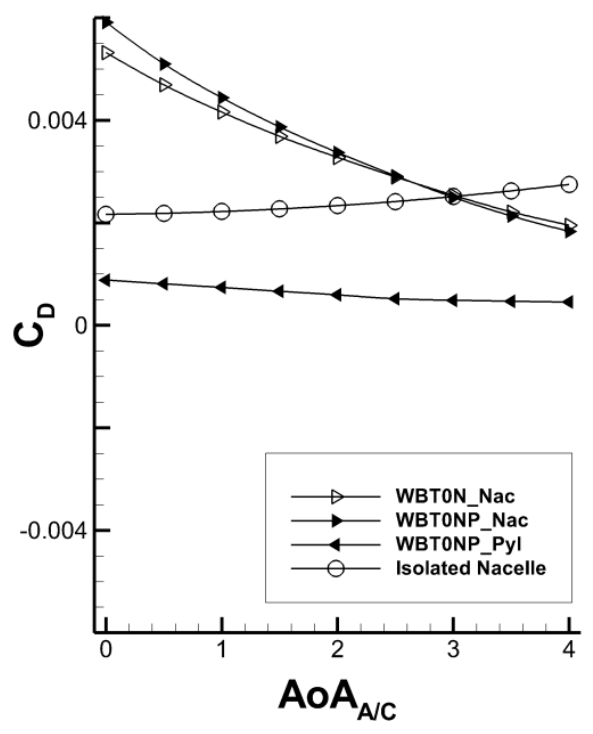

Figure17. Drag breakdown for nacelle and pylon components compared against drag coefficient for isolated nacelles.

As presented, the values of drag coefficient for each component depend on the other components present in the system. To isolate the effect of the nacelle from the effect of the pylon, the WBTON configuration was chosen for analysis at $R e=5 \times 10^{6}$ and $M=0.83$. The values of the drag coefficient for a component of the system change in relation to the drag coefficient of the same component analysed in isolation (Figure18). The presence of the airframe has amainly detrimental impact on the nacelle drag coefficient. Moreover, the observed trend for $C_{D \text { nac }}$ of a under-wing nacelle as function of AoAis the opposite of that for the isolated nacelle and it decreases with rising incidence. The action on the nacelle is accompanied by a beneficial reaction on the airframe side. The presence of the nacelle increased local suction in the front of the pressure side of the wing. As a result, the drag coefficient of the airframe, in the presence of the nacelle, reducedfor a given incidence in comparison to the clean wing computation (Figure18). Furthermore, by following the definition of the aerodynamic interference as a change of the flow due to the presence of other components1, an interference drag coefficientcan be defined as the change in drag coefficient due to aerodynamic interference foracomponent for a given aircraft 
angle of attack (equation (7)). The drag values have to be compared at appropriate angles of attack as the nacelle is installed with a pitch up angle of $A o A_{\text {inst }}=1.5^{\circ}$ relative to the aircraft defined AoA. The angle of attack for the installed nacelle decomposes to $A o A_{N a c}=A o A_{A / F}+$ $A o A_{\text {inst }}+A o A_{\text {upwash }}$. For the sake of the comparison, the values of drag for an isolated nacelle are taken as theaircraft angle of attack increased by the geometrical angle of installation (AoA $\left.A_{\text {inst }}\right)$, as the local wing upwash angle was determined to be negligiblefor thetested condition. In the tested configuration the average flow angle that enters the engine was only $-0.2^{\circ}$ from the nacelle angle of attack.

$C_{D \text { component interf }}\left(A o A_{A / F}\right)=\left[C_{D(\text { component in the system })}-C_{D(\text { component alone })}\right]_{A o A_{A / F}=\text { const }}$

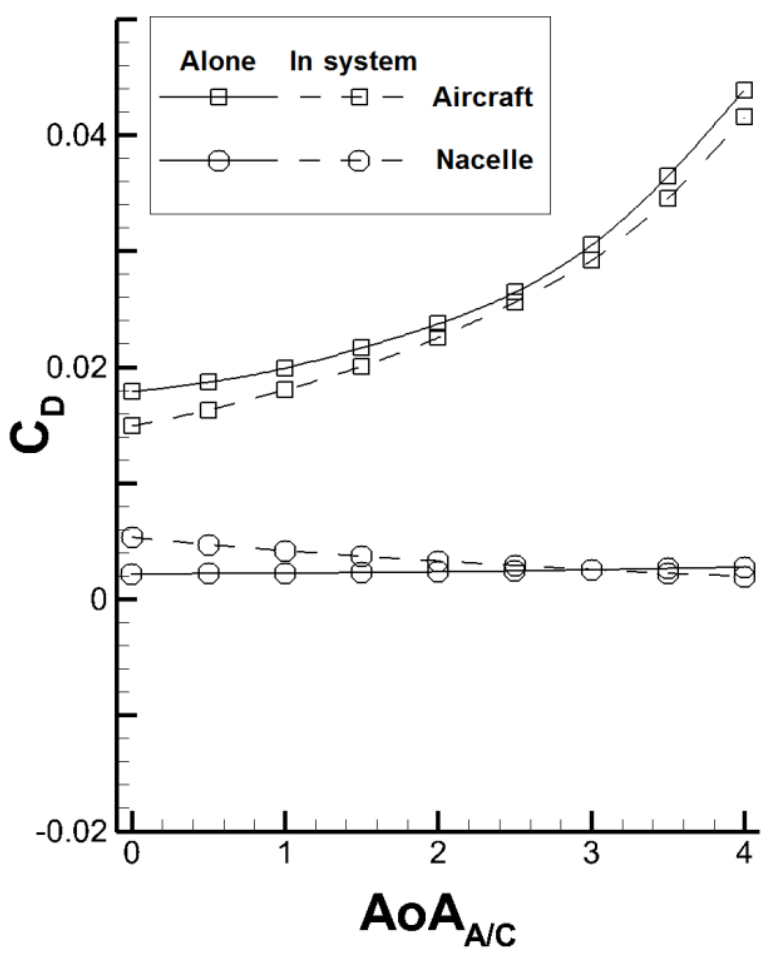

Figure18. Comparison of drag coefficient as a function of aircraft AoA for airframe (WBT0) and nacelle on their own and as component of WBTON configuration; $R e=5 x 10^{6} ; M=0.83$. 
The values of interference drag were computed for a range of angles of attack for the nacelle and for the airframe, where the aircraft consists of wing, body and tail (Figure19). The detrimental effect of the aerodynamic interference on the nacelle is observed. The greatest penalty $C_{D \text { nacinterf }}=+32 d c$ is observed at zero incidence and the impact reduces down to zero at an $A o A=3^{\circ}$. Although a small beneficial effect is observed at higher incidence, the magnitude of the effect is below $5 \mathrm{dc}$. At zero incidence, the presence of the wing reduced the suction on the forward facing portion of the nacelle forebody and thus increased the nacelle drag for the installed configuration. Meanwhile, the viscous drag of the installed nacelle is the same as the viscous drag on the isolated nacelle, and the nacelle MFCR had increasedslightly due to installation by $\triangle M F C R=+0.01$. At higher incidences, the beneficial effect of the wing pressure field on the nacelle afterbody becomes dominant, thus the drag coefficient for the wing-installed nacelle reduces. While the nacelle is mostly negatively affected by the presence of the airframe, the airframe is subject to beneficial aerodynamic interference. At zero incidencea benefit of $C_{D A / F \text { interf }}=-29 d c$ is observed. The benefit decreases with incidence up to $C_{D A / F \text { interf }}=$ $-9 d c$ at $A o A=2.5^{\circ}$ and increases again for greater incidence. Overall, the aerodynamic interference is the result of two competing effects such as the changes mainly in the pressure field on the nacelle and the airframe. For the current case, it is the benefit on the airframe and the penalty on the nacelle that result in an overall benefit $C_{D \text { tot interf }}=-4 d c$ on the aircraft drag at $A o A=2.5^{\circ}$. For clarity, this interference benefit is different from the overall installation penalty, which was defined in the section 'Effects of through flow nacelle' and for this case is approximately 20 drag counts. The interference effects on the airframe and the nacelle are predominantly the result of the pressure field that acts on the surfaces. The effect on the nacelle is the most notable one. The interference on the nacelle is the result of the pylon interaction with a suction peak at the nacelle leading edge, as well as the exposure of the fancowl afterbody $\left(\xi / L_{N a c}>0.7\right)$ to the wing pressure field (Figure 20). The contours of pressure coefficient for the isolated nacelle (Figure 20a)) were compared with the nacelle exposed to the wing pressure field (Figure 20b)). The outboard side $\left(\Theta>0^{\circ}\right)$ had a smooth distribution of the pressure coefficient 
(Figure 20b)) and it was broadly similar to the pressure distribution on the isolated nacelle (Figure 20a)). Meanwhile, the inboard side $\left(\Theta<0^{\circ}\right)$ had greater values of pressure coefficient at $\Theta=-30^{\circ}$ due to the exposure to the wing pressure field, as compared to the isolated nacelle. The effect diminished beyond $\Theta<-60^{\circ}$ and is relatively undisturbed on the bottom side of the nacelle.

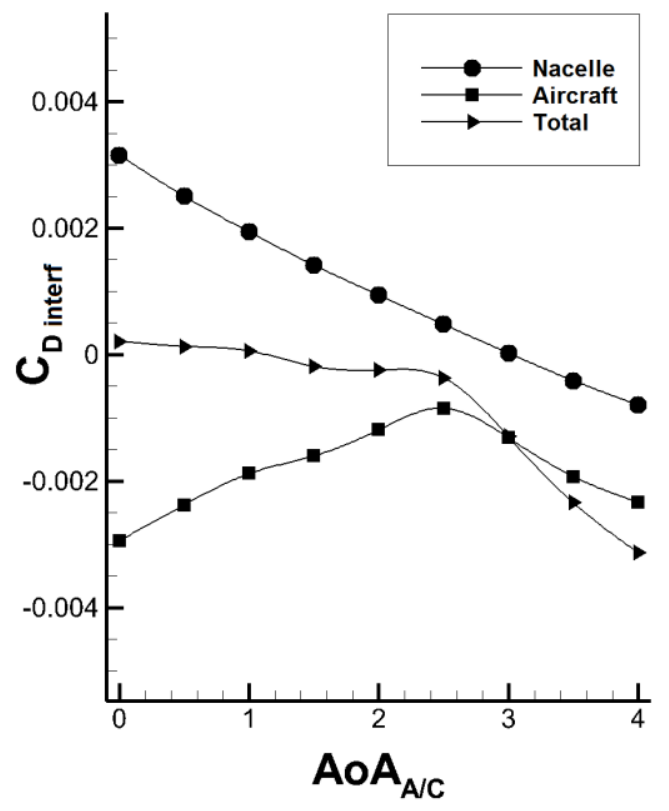

Figure19. Aerodynamic interference $\left(C_{D \text { interf }}\right)$ as a function of aircraft AoA $\left(A o A_{A / C}\right)$ for airframe, nacelle and total aerodynamic interference; $R e=5 x 10^{6} ; M=0.83$. 
a)

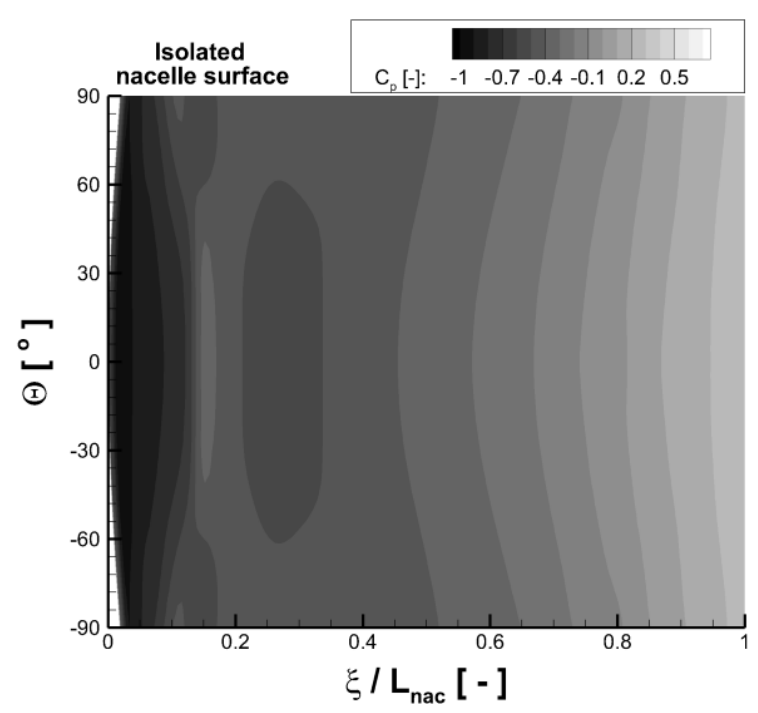

b)

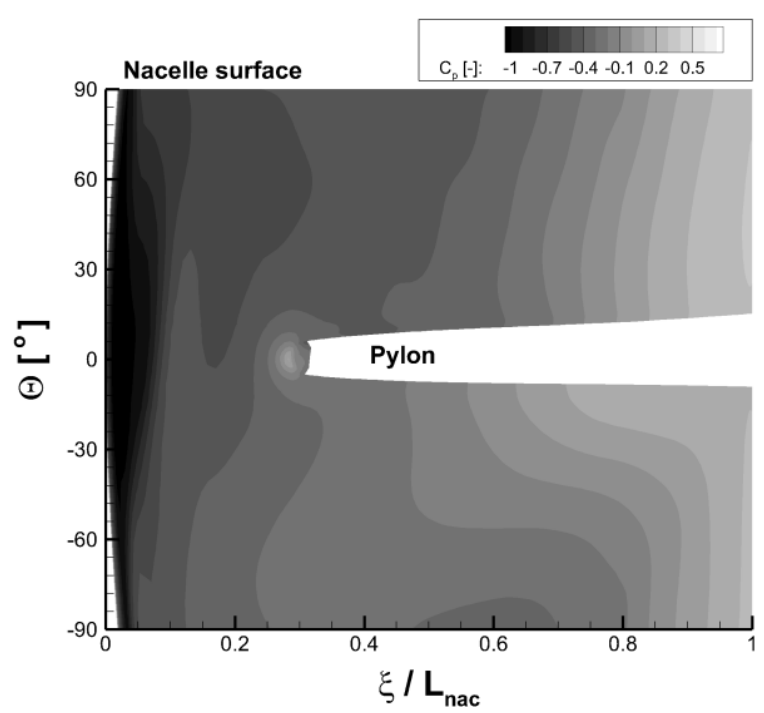

Figure 20. a) Contours of pressure coefficient $\left(C_{p}\right)$ for the top section of the isolated nacelle $(R e=$ $5 x 10^{6} ; M=0.83 ; A o A \approx 2.5^{\circ}$ ) compared with $b$ ) the top section of the nacelle exposed to the wing pressure field; fine mesh for WBTONP configuration, $R e=5 x 10^{6} ; M=0.83 ; A o A=2.5^{\circ}$.

\section{Conclusions}

Substantial progress has been made in the development of the underpinning tools and knowledgeto enable the preliminary design assessment of combined engine-airframe configurations. The main focus of this work is on developing some of the elements that are required to evaluate the impact of engine installation on the overall aircraft performance across the flight envelope. The isolated nacelle modelling criteria have been identified and the assessment of intake performance descriptors such as critical mass flow capture ratio and drag rise Mach number has been established. The effect of the installation of a throughflow nacelle on a transonic civil transport has been considered and the performance of a CFD method to determine theinterference aerodynamics has been assessed. Generally good agreement has been observed between experimental and numerical results for the quantification of installation 
effects. The numerical results were used for a breakdown of the drag and for the quantification of aerodynamic interference for the aircraft components.

\section{Acknowledgments}

The authors would like to thank J. C. Vassberg for making available the geometry of the Common Research Model including the throughflow nacelles.

\section{Funding}

Mr. Stańkowski's doctoral studies were partially funded by Rolls-Royce plc.

\section{References}

1. Advisory Group for Aerospace Research and Development (AGARD).Special course on Subsonic/Transonic Aerodynamic Interference for Aircraft. Report for NATO Science and Technology Organization.Report No. AGARD-R-712, January 1983.

2. Lee EE and Pendergraft Jr OC. Installation effects of long-duct pylon-mounted nacelles on a twin-jet transport model with swept supercritical wing. Report forNASA. Technical paper No. 2457, December 1985, Langley Research Centre, Hampton, Virginia.

3. Carlson JR andCompton WB.An experimental investigation of Nacelle-pylon installation on an unswept wing at susbonic and transonic speeds.Report forNASA. Technical paper No. 2246,February 1984, Langley Research Centre, Hampton, Virginia.

4. Hoheisel H.Aerodynamic aspects of engine-aircraft integration of transport aircraft,Aerospace science and technology,1997, Vol. 1, No 7, pp. 475-487.

5. Brodersen OComputation of Engine-Airframe Installation Drag,MEGAFLOW - Numerical Flow Simulation for Aircraft Design, edited by Kroll, N., Fassbender, J.,Vol. 89, Springer, Berlin, 2005. pp. 151-161. 
6. Rossow CC et al. Investigations of Propulsion Integration Interference Effects on a Transport Aircraft Configuration. In:AIAA/SAE/ASME/ASEE 28th Joint Propulsion Conference and Exhibit., Nashville (Tennessee), United States,6-8 July 1992, Paper No. AIAA 92-3097.

7. vonGeyr HF and Rossow CC.A correct thrust detemination method for turbine powered simulatiors in wind tunnel testing. In:41st AIAA/ASME/SAE/ASEE Joint Propulsion Conference \& Exhibit, Tuscon (Arizona), United States, 10-13 July 2005, Paper No. AIAA 2005-3707, pp. 1-12.

8. Frink NT. 2nd AIAA CFD Drag Prediction Workshop.Online Referencing,http://aaac.larc.nasa.gov/tsab/cfdlarc/aiaa-dpw/Workshop2/workshop2.html (2003 accessed 10.04.2015

9. Vassberg JC. et al. Development of a Common Research Model for Applied CFD Validation Studies, ReportNo. AIAA.2008-6919, Boeing Company, Huntington Beach.

10.Rivers MB and Dittberner A. Experimental investigations of the NASA Common Research Model in the NASA Langley National Transonic Facility and NASA Ames 11-Ft transonic wind tunnel (invited). In: 49th AIAA aerospace sciences meeting including the new horizons forum and aerospace exposition, Orlando, Florida, US, 4-7 January 2010, AIAA.2011-1126.

11. Rivers MB. Experimental investigation of the NASA Common Research Model (Invited). In:28th AIAA Applied Aerodynamics Conference, Chicago, Illinios, US, 28June - 1 July 2010, AIAA.2010-4218

12. Langley MJ. The design of axisymmetric cowls for podded nacelles for high by-pass ratio turbofan engines. ReportR\&M No. 3846, Aeronautical Research Council, 1979.

13. Roache PJ.Perspective: a method for uniform reporting of grid refinement studies.Journal of Fluids Engineering, Vol. 116, No. 3, 1994, pp. 405-413.

14.Zilliac GG Pulliam TH and Rivers MB. Comparison of the measured and computed skin friction distribution on the Common Research Model, In:49th AIAA aerospace sciences meeting including new horizons forum and aerospace exposition, Orlando, Florida, US, 4-7 January2011, AIAA.2011-1129 
15. Balakrishna S. Analysis of Common Research Model Dynamic Data, In:49th AIAA Aerospace sciences meeting including the new horizons forum and aerospace exposition, Orlando, Florida, US, 4-7 January 2011, AIAA.2011-1127

16. Frink NT, 4th AIAA CFD Drag Prediction Workshop.Online Referencing,http://aaac.larc.nasa.gov/tsab/cfdlarc/aiaa-dpw/Workshop2/workshop2.html (2003 accessed 10.04.2015

17. Tinoco EN, Levy D and Brodersen O. DPW-IV Summary of Participants Data, In:AIAA 4th CFD Drag Prediction Workshop, San Antonio, Texas, 2009.

18. Menter FR. Two-equation eddy-viscosity turbulence models for engineering applications, AIAA Journal, Vol. 32, No. 8, 1994, pp. 1598-1605.

19.ESDU. Introduction to the Measurement of Thrust in Flight (Air Breathing Ducted Flow Engines),Report ESDU-69006, ESDU. 1981.

20.Ministry-Industry Drag Analysis Panel (MIDAP) Study Group, Guide to in-flight thrust measurement of turbojets and fan engines.Report for NATO Science and Technology Organization.ReportNo. AGARD-AG-237, 1979.

21.Christie, R., Ramirez, S. and MacManus, D. G., "Aero-engine installation modelling and the impact on overall flight performance", In: Advanced Aero Concepts, Design and Operations Conference, 27 June 2014, Bristol, United Kingdom.

22. Destarac, Daniel. "Far-field/near-field drag balance and applications of drag extraction in cfd." VKI LectureSeries 2 (2003): 3-7.

23. Malouin, B., Gariepy, M., Trepanier, J.-Y. and Laurendeau, E., "Engine pre-entry thrust and standard netthrust evaluation based on the far-field method." Aerospace Science and Technology 45 (2015): 50-59. 


\section{Appendix I}

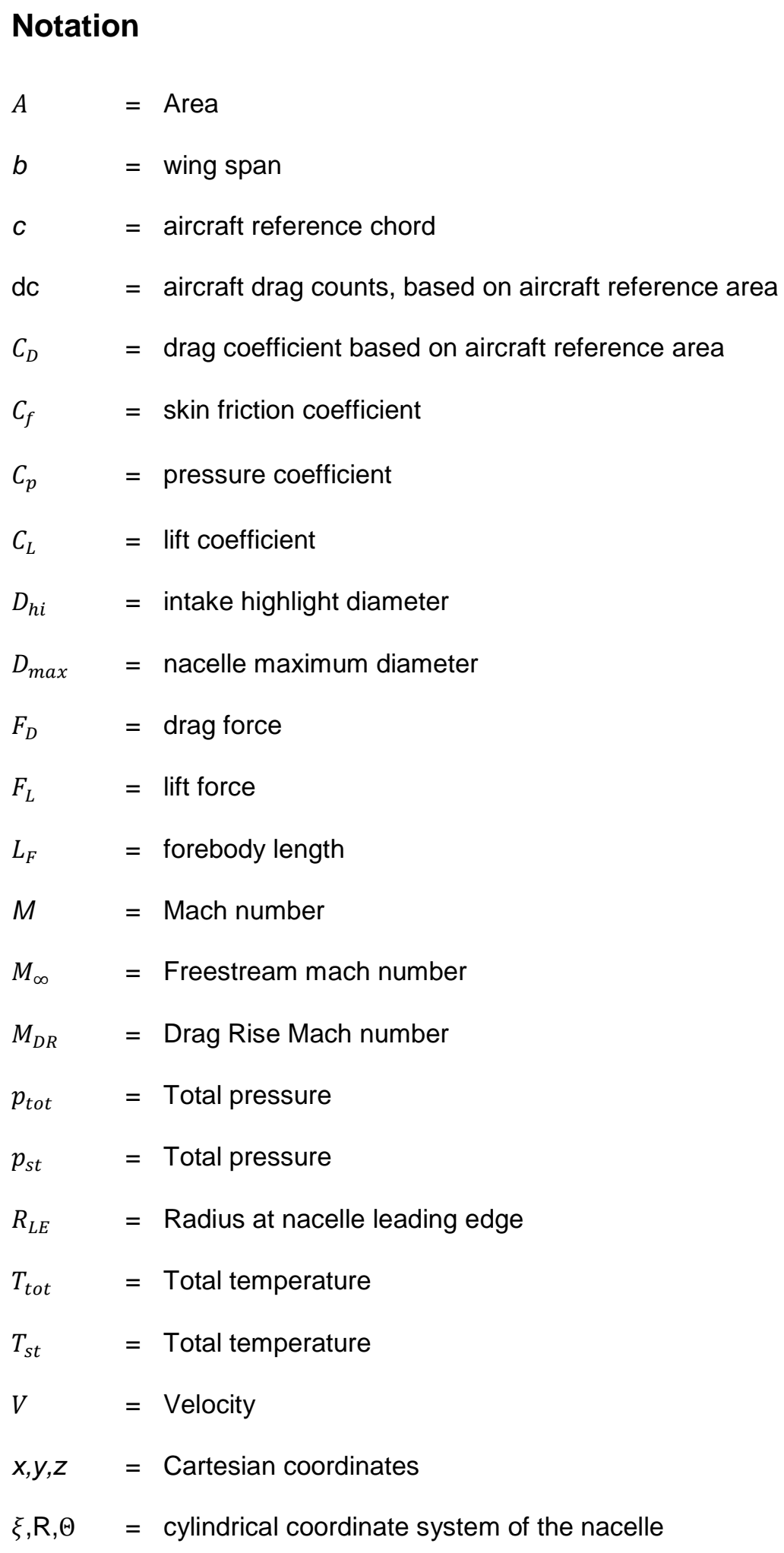




$$
\begin{array}{ll}
\phi & =\text { Force in the drag domain } \\
\theta & =\text { Force in the thrust domain } \\
\rho & =\text { mass density } \\
\eta \quad & =\text { non-dimensional spanwise position on the wing } \\
\omega & =\text { Vorticity }
\end{array}
$$

\section{Acronyms}

$$
\begin{array}{ll}
A / C & =\text { Aircraft, as Airframe and nacelle } \\
A / F & =\text { Airframe, as fuselage, wing and tail } \\
A O A & =\text { Angle of Attack } \\
A R A & =\text { Aeronautical Research Association } \\
C F D & =\text { Computational Fluid Dynamics } \\
C R & =\text { intake Contraction Ratio } \\
C R M & =\text { NASA Common Research Model } \\
\text { FF } & =\text { Fan face } \\
\text { MFCR } & =\text { mass flow capture ratio } \\
\text { TE } & =\text { Trailing edge } \\
T F N & =\text { Through Flow Nacelle } \\
W B & =\text { Wing Body } \\
W B N P \quad=\text { Wing Body with Nacelle and Pylon } \\
W B T O \quad=\text { Wing Body with } 0^{\circ} \text { inclined Tailplane } \\
W B T O N P=\text { Wing Body Tail with Nacelle and Pylon }
\end{array}
$$


2016-02-24

\title{
Aerodynamics of aero-engine installation
}

\author{
Stankowski, Tomasz P.
}

Sage Publications

pÿTomasz P StaDkowski, David G MacManus, Christopher TJ Sheaf and Robert Christie.

Aerodynamics of aero-engine installation. Proceedings of the Institution of Mechanical

Engineers, Part G: Journal of Aerospace Engineering, December 2016, vol. 230, no. 14, pp2673-2692

http://dx.doi.org/10.1177/0954410016630332

Downloaded from Cranfield Library Services E-Repository 